\title{
Bioelectronics with Two-dimensional Materials
}

\author{
Pilgyu Kang ${ }^{1}$, Michael Cai Wang ${ }^{1}$ and SungWoo $\operatorname{Nam}^{1,2 *}$ \\ ${ }^{1}$ Department of Mechanical Science and Engineering, University of Illinois at Urbana-Champaign, \\ Urbana, IL 61801 USA \\ ${ }^{2}$ Department of Materials Science and Engineering, University of Illinois at Urbana-Champaign, Urbana, \\ IL 61801 USA \\ *corresponding authors: pkang7@illinois.edu; swnam@illinois.edu
}

\begin{abstract}
In this article, we review the emerging field of bioelectronics with two-dimensional (2D) materials. Recently, 2D materials including graphene, transition metal dichalcogenides (TMDs), and other elementary 2D crystals, have emerged as functional materials in bioelectronic applications benefitting from their superior electrical, optical, and mechanical properties compared to conventional bulk semiconductor and metallic materials. The 2D dimensional materials have advanced bioelectronics by allowing easy integration due to their atomic thinness, biocompatibility, mechanical flexibility and conformity. Electronics with 2D materials have thus far enabled various applications in chemical, biochemical and neurobiological sensing. Here we review bioelectronics with a variety of $2 \mathrm{D}$ materials including graphene, a popular material in the recent decade, as well as other emerging 2D materials such as TMDs including $\mathrm{MoS}_{2}, \mathrm{MoSe}_{2}, \mathrm{WSe}_{2}$, and $\mathrm{WS}_{2}$. First, we discuss the unique properties of 2D materials which make them suitable for bioelectronic applications. Next we highlight transduction and detection mechanisms of 2D material-based bioelectronic systems, which include field-effect transistors, nanopores, multi-electrode arrays, and optical resonators. Lastly, we highlight current ongoing efforts to enable 2D materials to be even more effective in performance and sensitivity for biointerfacing.
\end{abstract}

Keywords: Bioelectronics, 2-dimensional materials, Graphene, Transition metal dichalcogenides 


\section{Table of Contents}

1. Introduction

1.1. 2D Materials

1.1.1. Synthesis and Processing

1.1.2. Heterostructures of 2D Crystals

1.2. 2D Nanomembrane Materials

1.2.1. Ultrathin Nanomembranes

1.2.2. 3D Enveloping Membranes

2. Material Properties Required for Bioelectronics

2.1. Biocompatibility and Bioresorbability

2.2. Mechanical Flexibility and Stretchability

2.3. Shape Conformity

3. Detection/Sensing Mechanisms

3.1. Field-effect Transistor based Sensing Mechanism

3.2. Nanopore-based Sensing Mechanism

3.3. Multi-electrode Array based Sensing Mechanism

3.4. Optical Resonator-integrated Sensing Mechanism

3.5. Multi-functional Sensor Array based Sensing Mechanism

4. Fabrication Techniques for 2D Material based Bioelectronics

4.1. Patterning of Transistor Channels

4.2. Fabrication of Source/Drain and Gate Electrodes

4.3. Integration of Reference Electrodes

4.4. Functionalization

5. Sensing Applications

5.1. Chemical and Molecular Sensors

5.1.1. Molecular Sensors based on Amperometric/Conductometric Measurements

5.1.2. Field-effect Transistor based Sensors

5.1.3. 2D Material-integrated Optical Sensors

5.2. DNA Sequencing Technology based on 2D Material Nanopores

5.3. Neurobiological and Cardiac Sensors

5.3.1. Multi-electrode Arrays

5.3.2. Field-effect Transistors

5.3.3. Multi-functional Sensors

6. Remarks and Outlook

6.1. Strain Engineering of 2D Materials

6.2. 3D Topographic Engineering of 2D Materials

6.3. Hybrid Systems of Low-dimensional Materials and 2D Materials

6.4. Biodegradable Electronics

6.5. Challenges on 2D Materials based Bioelectronics

6.6. Future Directions of 2D Materials based Bioelectronics

Acknowledgements 


\section{Introduction}

Over the past decade, bioelectronics, where electronics are interfaced with biology and biotechnology,[1] have been a vibrant field of research through rapid developments in both fields of electronics and biomedical engineering. Bioelectronics integrates biological materials like proteins, antibodies, DNA, and cells with electronic elements such as field-effect transistors (FETs),[2, 3] electrodes [4-9] and electrode arrays,[10-18] and optical resonators [19-23] to realize functional biosensing devices (Fig. 1a).[24-26] Such bioelectronic devices detect and monitor biological interactions and physiological signals by electrical/optical readouts as a means of the change in current, potential, conductance, or resonant frequency. Recently efforts have been made to develop high performance bioelectronic devices by utilizing advanced materials, in particular, two dimensional (2D) materials.[1, 27-45]

2D materials including graphene[29] and transition metal dichalcogenides (TMDs)[28] have attracted significant attention because of their outstanding electrical,[2, 29] optical,[46, 47] and mechanical properties,[48, 49] enabling performance improvements of bioelectronic devices.[46, 47] In addition, atomically thin 2D materials compared with bulk materials inherently lead to high sensitivity attributable to their large surface-to-volume ratio.[36, 50] Emerging 2D materials including graphene and TMDs $\left(\mathrm{MoS}_{2}\right.$, and $\mathrm{WS}_{2}$ monolayers, etc.) have demonstrated several advantages for developing implantable bioelectronic systems suitable for in vivo environments, serving requirements for biocompatibility $[50,51]$ and bioresorbability,[52] mechanical flexibility and deformability[53, 54] and shape conformity.[55,56] Conventional bulk materials including metals and semiconductor (silicon and germanium) have been widely used mainly because of their established and mature capability to construct metal electrode arrays or field-effect devices as transducers for bioelectronic sensing, [56, 57] in addition to easy integration with auxiliary detection units such as amplification and signal-processing units for practical devices.[58] They have also provided flat and homogeneous substrates to conjugate probing biomolecules with improved interfacial properties. However, conventional materials have been limited to 
realize high performance bioelectronic devices and meet requirements for in vivo environments. On the contrary, 2D materials have demonstrated the capability to serve such needs with the aforementioned advantages.

In this regard, this review article highlights recent developments of bioelectronics with 2D materials as well as 2D nanomembrane materials constructed with conventional silicon materials and other transient electronic materials.[31, 59, 60] First, we discuss the recent developments of 2D materials and 2D nanomembrane materials, including the variety of different materials and their respective synthesis and processing approaches [61] for the integration with sensing electronic elements. [62] In the following, we describe the requisite material properties to develop bioelectronic sensing systems for implantable devices and functional devices for in vivo applications. Next we review probe and sensing technologies for multiplexing as well as high signal-to-noise ratio and sensitivity; technologies covered include 1) FETs, 2) nanopores, 3) multi-electrode arrays (MEAs), 4) optical resonators and 5) multifunctional sensing arrays. Furthermore, we emphasize various applications that have been developed in a wide range of fields, including 1) chemical and environmental sensing, 2) biochemical sensing and 3) neurobiological/cardiac sensing (both in vivo and in vitro). Lastly we conclude with outlooks on recent efforts to develop three dimensional (3D) topographical materials from $2 \mathrm{D}$ materials for improved performance and strain-tolerant, shape conformal devices, as well as the hybrid approaches that integrate other low dimensional nano-materials with 2D materials.

\subsection{D Materials}

In this section, we discuss synthesis of $2 \mathrm{D}$ materials. We focus on graphene, considering that graphene is a superlative material in regards to its highest mechanical strength and unparalleled electronic and optical properties. Other 2D crystals include graphene-related materials (graphene oxide, hexagonal boron nitride (hBN), BCN, fluorographene) and semiconducting 2D TMDs $\left(\mathrm{MoS}_{2}, \mathrm{WS}_{2}, \mathrm{MoSe}_{2}\right.$, and $\mathrm{WSe}_{2}$ monolayers) (Fig. 1b).[28] Heterostructures made by vertically stacking different 2D crystals have 
also been developed, and such heterostructures typically revolve around graphene because of the unmatched properties. For graphene, different synthesis methods result in distinct characteristics and we discuss these distinctive properties resulting from the preparation methods. In addition, we discuss the important processes of transfer and patterning, which are necessary for device developments. Lastly we briefly comment on assembly techniques of emerging heterostructures of different $2 \mathrm{D}$ crystals. These discussions will provide insights about optimal synthesis and processing techniques for achieving bioelectronic devices with specific performance and design.

\subsubsection{Synthesis and Processing}

Single layer graphene with high quality in structural integrity is obtainable by mechanical cleavage of highly oriented pyrolytic graphite.[2] Mechanically exfoliated graphene exhibits excellent electronic characteristics including high carrier mobility $\left(20,000 \mathrm{~cm}^{2} \cdot \mathrm{V}^{-1} \mathrm{~s}^{-1}\right),[2,29]$ high carrier density $\left(10^{13} \mathrm{~cm}^{-2}\right)$ [63] as well as high optical transparency ( $2.3 \%$ absorption in visible).[47] These outstanding properties have been useful in many research on electronic/optoelectronic devices because of low structural defects. However, low throughput and inability to produce large size graphene have been considered drawbacks for mechanically exfoliated graphene, limiting practical uses. Notably, typical size limit of graphene obtained by mechanically exfoliation is a few tens of micrometers.

Chemical vapor deposition (CVD) onto metal substrates such as copper $(\mathrm{Cu})$, nickel (Ni), palladium (Pd), iridium (Ir), and ruthenium $(\mathrm{Ru})$ is considered an inexpensive and promising synthesis method for large-scale, high quality graphene. Among these substrates, copper foil has served uniform single layer graphene with excellent characteristics such as the Hall mobility of $7350 \mathrm{~cm}^{2} \cdot \mathrm{V}^{-1} \mathrm{~s}^{-1}$ at $6 \mathrm{~K}$ and $\sim 5,100 \mathrm{~cm}^{2} \cdot \mathrm{V}^{-1} \mathrm{~s}^{-1}$ at $295 \mathrm{~K}$ and large size (up to 30 inches).[46] However, defects and impurities are introduced in CVD graphene. In addition, a transfer process is needed for CVD graphene from the synthesis substrate to a target substrate for device fabrication. This transfer process introduces additional impurities, degrading the properties of CVD graphene. Graphene synthesis on copper substrate (Fig. 1c) 
involves the decomposition of methane gas at $\sim 1000^{\circ} \mathrm{C}$, during which doping of graphene is also possible by using nitrogen and boron gases. The doping capability is an advantage of CVD growth as compared with the mechanical exfoliation method. It is also possible to obtain large graphene domain sizes, led by increased $\mathrm{Cu}$ grain sizes with pre-treatment of the copper substrate.

Graphene transfer is carried out by coating a protective polymer layer on top of a graphene film, such as polydimenthysiloxane (PDMS) or polymethylmethacrylate (PMMA). This is followed by etching the growth substrate (copper) with $\mathrm{FeCl}_{3}, \mathrm{Fe}\left(\mathrm{NO}_{3}\right)_{3},\left(\mathrm{NH}_{4}\right)_{2} \mathrm{SO}_{8}$, or $\mathrm{CuCl}_{2} . \mathrm{FeCl}_{3}$ is widely used because of etching process without gaseous products. Etching the growth substrate with the protected graphene film results in a free-standing graphene floating on the solution. The polymer coating allows handling of graphene film onto a desired substrate and is removed after the transfer process. Scalable transfer of CVD-grown graphene onto a flexible plastic substrate ( 30 inches in diagonal) was shown by using the roll-to-roll transfer method [46] for which a thermal release tape was used as a supporting polymer layer. Transfer can also be carried out by using an elastomeric stamp such as PDMS.[64] Raman spectroscopy is widely used to examine defects in graphene, characterized with the intensity of D band at $\sim 1,350 \mathrm{~cm}^{-1}$. Other distinctive peaks of graphene include $2 \mathrm{D}$ band at $\sim 2,660 \mathrm{~cm}^{-1}$ and $\mathrm{G}$ band at $\sim 1,580 \mathrm{~cm}^{-1}$.[65]

CVD growth of graphene is one of the most promising methods for obtaining high quality, large area graphene. Although wrinkles and grain boundaries are minimal in graphene grown on $\mathrm{Cu}$ substrate, a reliable and scalable transfer method is needed to realize high performance devices, for example, with mechanical flexibility/stretchability. Notably, synthesis methods, doping, and transfer methods determine the properties of graphene. Previous reviews offer further comprehensive information on graphene preparations and the resultant properties.[61, 62, 66, 67] Graphene patterning may be carried out by standard photolithography, followed by etching unwanted region of graphene with plasma treatment. Ebeam lithography is used for producing patterns on the scale of $\sim 10-100 \mathrm{~nm}$.

\subsubsection{Heterostructures of 2D Crystals}


There are various $2 \mathrm{D}$ crystals obtained by mechanical exfoliation from bulk crystals to atomically thin layers. Many classes of layered materials have been reported, including graphene related materials (graphene, hBN, and graphene oxide), semiconducting dichalcogenide (molybdenum and tungsten based), metallic dichalcogenides (niobium and tantalum based), layered semiconductors (GaSe, GaTe, and $\mathrm{Bi}_{2} \mathrm{Se}_{3}$ ), and so on. They each have their own distinctive properties, for example, a direct band gap for monolayer $\mathrm{MoS}_{2}$ and indirect band gap for multilayer or bulk $\mathrm{MoS}_{2}$. (see also [28] for more comprehensive discussion). Reducing dimensionality to one or a few layers may enable broad electronic/optoelectronic applications benefiting from the different electronic and optical properties from the bulk. Mechanical properties such as flexural strength (or bending rigidity) is also modulated as a result of change in thickness of layered materials.[68] While mono- or bilayer graphene or $\mathrm{MoS}_{2}$ exhibit membrane-like behavior yielding negligible bending rigidity, they show plate-like behavior with increasing layer thickness.[68] Although 2D crystals are desirable when used exclusively on their own because of their unique properties as single layered materials, heterostructures obtained by stacking two or more layers of 2D materials are also gaining attention.[53,61, 69] Recently, the heterostructure of graphene-hBN was developed to investigate the electronic transport that undergoes electron-electron interactions between the electrically insulated layers of 2D crystals. On the other hand, such heterostructure systems may find potential in bioelectronic applications with enhanced performance as a biosensor for long term viability of sensing capability; hBN can be used as a protective/passivation layer or substrate.[70]

\subsection{D Nanomembrane Materials}

In addition to inherently atomically-thin 2D materials, ultrathin membrane materials in a mesh configuration have been researched in emerging fields of transient $[31,59,60]$ and stretchable electronics.[41, 64, 71] Such emerging fields have a wide range of applications in implantable and biodegradable electronics.[59, 72] The ultrathin membrane materials have been actively investigated, and 
we highlight nanomembrane materials in the form of small-scale 2D flexible sheets and 3D elastic membranes, respectively, with a focus on materials and design. While consistent and time-invariant operations have been a prerequisite for reliable performance in conventional electronics, researchers have recently put efforts to develop implantable and surgical devices with biodegradability. Bio-integrated systems comprises many constitutive components including gate dielectric layer, metal interconnects, electrodes, substrates, and encapsulation layers for which functional materials have to be properly selected and designed to attain the ultra-thin and mesh forms.[71] The design and materials enable the bioelectronic systems' conformal integration with biological tissue, ensuring minimal stresses on the biological tissue and sensing stability.[55] The bioelectronic systems with the nanomembranes were limited to small-scale 2D flexible sheets. However, 3D elastic membranes have been recently shown by 3D printing, enabling an integumentary device with multifunctional sensors including strain gauge, electrical sensing and stimulation, $\mathrm{pH}$ sensors, and temperature sensors and heaters. [73]

\subsubsection{Ultrathin Nanomembranes}

Besides intrinsic 2D materials, materials and designs using conventional semiconductor materials such as silicon have realized two-dimensionality, led by an emerging field of transient electronics.[59, 60] The approach is to integrate biodegradable electronics that comprises a variety of electronic elements including substrates, interconnects, and electrodes for multifunctional sensors.[73, 74] Potential applications include biomedical devices, biodegradable environmental sensors, and disposable electronic devices.[73, 75] Notably, materials comprising the electronic components can be dissolved in biofluids by hydrolysis. [59] The materials used include silicon in a thin nanomembrane configuration, biodegradable polymers for substrates (such as poly lactic acid (PLA), poly lactic co-glycolic acid (PLGA), polycaprolactone (PCL), polyimide (PI), rice paper,[52] and silk fibroin [55]), organic electronic materials (such as bacterial cellulose membranes [74] and water soluble polymers [52]), inorganic semiconductor materials such as 5,5'-bis-(7-dodecyl-9H-fluoren-2-yl)-2,2'-bithiophene (DDFTTF) [32], 
$\mathrm{Si}$ and $\mathrm{ZnO}$ [31] and metals for interconnects and electrodes such as $\mathrm{Mg}, \mathrm{Fe}, \mathrm{Zn}$, and W.[31]Various approaches have been explored to develop electronics soluble in water as well as transient electronic device on biodegradable polymer substrates. $[32,59,72]$ Besides the materials, a specific nanomembrane configuration enables controlled transient behavior in water and biofluids by hydrolysis. The kinetics of Si nanomembranes has been extensively investigated for in vivo and in vitro conditions, where the materials undergo hydrolysis in biofluids and various aqueous solutions under varyious conditions $(\mathrm{pH}$, ionic concentrations, and temperatures).[31] These transient silicon-based materials including $\mathrm{Si}_{\text {and }} \mathrm{SiO}_{2}$ can serve high performance in essential electronic components of the integrated systems such as transistors, diodes, temperature sensors, and strain gauges.[60] The integrated electronic systems have been realized in small-scale 2D flexible sheets, enabling flexible and implantable electronics.

\subsubsection{D Enveloping Membranes}

High-density physiological recordings and stimulation needed in clinical research and applications have been a challenge by utilizing 2D nanomembranes systems, which are limited for shape conformity to an organ level because of a planar nature. Recently, a 3D integumentary device for cardiac measurements have been shown by $3 \mathrm{D}$ printing that shapes a $3 \mathrm{D}$ elastic membrane to entirely envelop the epicardial surface. The integumentary elastic membranes enable stable conformal contact for high density multiparametric and temporal sensing and mapping on the heart muscles. The multiparametric sensing and mapping were realized by integrated functional elements that include strain gauges, electrocardiogram (ECG) sensors and inorganic light-emitting diodes with silicon nanomembranes, $\mathrm{pH}$ sensors with iridium oxide pads and temperature sensors/heaters with Au resistors.[73] The demonstration was shown by ex vivo experiment with a rabbit heart. However, challenges remain to integrate communication, power supply, and control systems for obtaining implantable bioelectronic devices that will be utilized in clinical research and biomedical applications. 


\section{Material Properties Required for Bioelectronics}

2D materials and 2D nanomembranes provide unique material properties for bioelectronics applications. Here we highlight the materials properties that are advantageous to bioelectronic applications; a) biocompatibility and bioresorbability, b) mechanical flexibility and deformability, and c) shape conformity. We focus on the properties that revolve around bioelectronic sensing and implantable devices. The devices have potential not only in biomedical and clinical research applications, but also in stretchable and flexible electronics. Moreover, flexible electronics has great potential for wearable electronics as an integrated biosensing system that is conformal to the epidermis. Biocompatibility is a requirement for implantable devices, and recent biodegradable devices attained by materials and design [40] have been extensively investigated for the application. 2D nanomembranes have been also developed by 3D integumentary membrane devices with integrating multifunctional electronic and optoelectronic systems for sensing and stimulation to attain the mapping of responses with high spatial resolution at the organ level.

\subsection{Biocompatibility and Bioresorbability}

A wide range of nanomaterials including carbon-based materials, metals, and semiconducting materials have been widely used in biomedical research and biotechnologies. In particular, carbon-based nanomaterials including graphene, carbon nanotubes, and nanodiamonds have demonstrated increasing applications in bioelectronics not only because of outstanding properties such as electrical conductivity, mechanical robustness, and optical properties (broadband absorption and transparency),[29] but also because of their biocompatibility (Fig. 2a).[30, 34, 35, 38, 76, 77] Their biocompatibility has been discussed and reviewed recently by Pinto et al. [40] with a focus on the interfaces with bacteria and mammalian cells. The biocompatibility is dependent on intrinsic physical and chemical properties of the materials. Functionalization, synthesis and post-processing of materials can have an effect on biocompatibility [40]; The functionalization with biological molecules enhances biocompatibility, 
whereas residual metals from synthesis catalyst may cause cytotoxicity to interfacing with cells and bacteria. Biocompatibility of graphene-based materials chemically modified from graphene and graphene oxide (GO), as well as $\mathrm{MoS}_{2}$ has been extensively investigated [40, 50] in order to (1) increase cell growth and viability as well as (2) improve electrical properties for enhanced sensitivity. Notably, 2D materials such as $\mathrm{MoS}_{2}$ and $\mathrm{WS}_{2}$ have been deemed intrinsically biocompatible.[50, 51]

\subsection{Mechanical Flexibility and Stretchability}

Mechanical flexibility or stretchability [78] are necessary characteristics of the bioelectronic systems that are integrated conformably to biological tissues or entire organs such as skin, heart, and brain for broad applications in heath monitoring (e.g. wearable electronics), human-machine interfaces (e.g. bionics), and biomedical or clinical research (e.g. neurobiology and cardiology) (Fig. 2b). Such mechanically flexible devices enable conformal interfacing to the biological surfaces, enabling reliable monitoring of physiological signals over a long period of time. The stretchable devices mainly comprise of functional systems performing sensing and stimulation, and elastic substrate materials. The system design with appropriate materials has been a key strategy to minimizing strain concentration on the functional units and sustain applied strain by the flexible substrates.[78-80] In this regard, graphene-based bioelectronic systems have gained increasing interest as a main functional unit that can sustain strain by utilizing exceptional mechanical strength of the graphene, which is capable of sustaining up to $25 \%$ tensile strain.[48]

\subsection{Shape Conformity}

Conformal electronic systems are needed to construct stable interfaces between biological surfaces and functional electronic elements. At the cell or tissue level, the form of $2 \mathrm{D}$ sheets has sufficiently allowed reliable interfacing by using flexible and stretchable materials for a substrate. However, it has been challenging to conformably envelop an entire organ (especially such as a heart) to 
maintain a stable interface for spatiotemporally mapping and stimulating cardiac physiology without interfering cardiac muscle movements including contraction and relaxation. Moreover, high density multifunctional recording and accurate measurements and stimulations have been required for organs such as the brain and heart, and 3D integumentary membrane devices were demonstrated for such purposes by $\mathrm{Xu}$ et al.[73] $\mathrm{Xu}$ et al. achieved the 3D heart structure with 3D printing, and integrated multifunctional electronics (e.g. $\mathrm{pH}$, temperature, and strain sensors) and optoelectronic components onto 3D integumentary membrane. Such achievements have great potential in implantable devices especially in cardiology and neuroscience for biomedical and clinical research.

\section{Detection/Sensing Mechanisms}

In this section, we discuss transduction and detection mechanisms of diverse bioelectronic systems integrated with 2D materials, including FETs,[32, 36, 49, 51, 81-90] optical/electrical detection using nanopores,[25, 91-103] electrodes[4, 5, 9, 104, 105] and electrode arrays,[10-12, 14, 16-18, 106, 107] optical resonators,[19-23] and multifunctional sensor arrays.[73, 75] A bioelectronic sensing device is attained by integrating biological recognition probes (e.g., protein-enzymes, antigen/antibodies, DNA, neurons, and cells) with electronic sensing elements. The sensing elements transduce biological interactions with the probes to the readouts through electrical current, potential, conductance, impedance, and resonant frequency resulting from alternation of interfacial properties led by such interactions.

For example, graphene-based electronic sensing unit can be functionalized with linker molecules covalently bonded to graphene surface. The linker molecules enable detection specificity, promote signal transduction and provide amplification of sensing probes.[37] However, the covalent bonding scheme

changes electrophysical properties of graphene from $\mathrm{sp}^{2}$ to $\mathrm{sp}^{3}$ bonding, degrading electronic performance (i.e., reduced carrier mobility). On the other hand, non-covalent methods use electrostatic, van der Waals, hydrophobic interactions between coupling molecules and graphene surface. The non-covalent methods allows facile functionalization with physical adsorption of various molecules, but also yields non-specific 
adsorption. The non-specific adsorption can be solved by passivation which blocks non-functional sites using surfactants (e.g. Polysorbate 20) and stabilizing biomolecules (e.g. bovine serum albumin).

2D materials, especially graphene-based materials, have been extensively investigated because of advantages, such as high carrier mobility $\left(15,000 \mathrm{~cm}^{2} /(\mathrm{V} \cdot \mathrm{s})\right)$, sensitivity to local electrochemical perturbation, tunability of electrical properties (i.e., carrier density) by electrostatic gating,[82, 87] opening zero band-gap by chemical doping $[19,108]$ and large, homogenous detection area attributable to high surface-to-volume ratio $\left(2600 \mathrm{~m}^{2} / \mathrm{g}[24,36]\right)$. In particular, the graphene surface enables uniform and effective functionalization,[109] biocompatibility enabling cell adhesion and growth,[110] optical transparency enabling optoelectronic stimulation [15, 33, 111] and electrical measurements. In the following section we discuss the respective detection mechanisms based on 2D materials.

\subsection{Field-effect Transistor based Sensing Mechanism}

Graphene-based FET biosensor is based on a configuration similar as the one shown in Figure 3a of a solution-gated FET device. Most importantly, effective gating is obtained by using an electrolyte, and the conductance of a graphene FET is modulated by gate potential between a reference electrode and grounded electrode (drain). In experiment, source-drain current $\left(I_{\mathrm{SD}}\right)$ is measured as a function of the gate voltage $\left(V_{\mathrm{G}}\right)$, showing a minimum source-drain current at a finite gate-voltage (Dirac point). In general, the sensing of target biomolecules is dominated by the electrostatic gating effect [112-114] in combination with Schottky barrier effect.[114-117] Such sensing mechanism was elucidated by Heller et al. with extensive experimental studies.[118] In electrostatic gating effect, biomolecule adsorption induces an electrostatic disturbance that leads to a change in transistor conductance. In other words, adsorbed charged biomolecules induces doping in the graphene that leads to a shift of the Dirac point.[37, 108, 119, 120](Fig. 3a,b) On the other hand, in the Schottky barrier mechanism, biomolecules absorbed on the metal contact causes a change of the difference between workfunctions of the metal contacts and conductive channel that leads to an asymmetric conductance change in p- and n- branches of a $I_{S D^{-}} V_{G}$ 
curve. Importantly, it was shown that the electrostatic gating effect becomes predominant over the Schottky barrier effect by suppressing inconsistent Schottky barrier effect with the passivation of the conductive channel-metal contact. Other suggested mechanisms include the effects of changes in carrier mobility [121, 122] and reduced gate efficiency.[123, 124] Adsorbed biomolecules on sensing location induce scattering of charge carriers that leads to reduced carrier mobility. Relatively low permittivity of adsorbed biomolecules, compared to that of the electrolyte, decreases the gate capacitance, leading to the reduced gate efficiency. However, these changes in carrier mobility and gate coupling were shown to be negligible, compared with the effects of the electrostatic gating.[118]

It is important to consider the Debye length for biological applications of $2 \mathrm{D}$ material FET devices. [87] The Debye length is a distance in which the target and probe biomolecules are electrically screened by surplus ions in an electrolyte. The Debye length $\left(\kappa^{-1}\right)$ is dependent on the buffer solution's ionic concentration, temperature and dielectric constant. It is defined as $\kappa^{-1}=\left[\varepsilon_{r} \varepsilon_{0} k_{B} T /\left(2 N_{A} e^{2} \mathrm{I}\right)\right]^{1 / 2}$ for a monovalent electrolyte, where $\varepsilon_{0}$ is the vacuum permittivity, $\varepsilon_{r}$ is the dielectric constant, $k_{B}$ is the Boltzmann constant, $T$ is temperature in Kalvin, and $N_{A}$ is the Avogadro number, $e$ is the elementary charge, and I is ionic concentration of an electrolyte in molar (M).[125] A typical buffer solution, 5-10 $\mathrm{mM}$ of a Phosphate-buffered saline (PBS) solution used in biosensing experiments, yields $\sim 5 \mathrm{~nm}$ of the Debye length at room temperature. Most importantly, charged biomolecules, at a distance larger than the Debye length from the FET device surface, rarely affect the mobile charges in a FET channel; the interactions between target and probe biomolecules need to be occurred within the Debye length, and the size of a probe biomolecule is required to be smaller than the Debye length. In these regards, the ionic strength of a buffer solution and size of interacting biomolecules need to be considered to account for detection limit.

This field effect mechanism allows not only electrochemical and electrical sensing of DNA,[83] biomolecules,[87, 126] and cells,[11, 81] but also electrical sensing of $\mathrm{pH}$ levels. We discuss these applications in more detail in Section 5. 


\subsection{Nanopore-based Sensing Mechanism}

In this section, we discuss the mechanism of nanopore-based sensing using $2 \mathrm{D}$ materials, specifically graphene and $\mathrm{MoS}_{2}$ monolayer with focus on the application of DNA analysis.[45, 98, 127, 128] Nanopore-based sensing employs a nanometer sized aperture on a thin membrane. An applied electric potential on the membrane separating two compartments filled with conductive electrolyte drives charged molecules to pass through a nanopore, and passage of charged molecules modulates the ionic current across the nanopore (Fig. 3c,d).[97, 127] Such current provides information about molecular dynamic motion and structure. The nanopore-based sensing is useful in applications of DNA sequencing in that it enables label-free, high throughput single molecule analysis. To be more specific, as a single strand of DNA passes through the nanopore, the ion current can change depending on the type of nucleotide or base that is translocating through the nanopore. Thus, recording the change of the ionic current allows the determination of the base sequence in the DNA molecule. Nanopore-based sensing unit is further integrated with optical [96, 98] or local electrical readout [99] techniques enabling highthroughput.

Graphene is advantageous for DNA sequencing compared with other solid-state nanopore membrane materials such as silicon nitride $(\mathrm{SiN})$ because of its thinness; the thickness of a single-layer graphene is comparable to the spacing $(0.32-0.52 \mathrm{~nm})$ between nucleotides,[129] the subunits of nucleic acids like DNA and RNA. On the other hand, due to cracks and defects of graphene membrane resulting in poor insulating property, the noise of the ionic currents may be larger by several orders of magnitude compared with SiN.[101, 130] However, a thin layer ( a few nanometers) of titanium dioxide $\left(\mathrm{TiO}_{2}\right)$ over the graphene membrane showed considerable reduction in the noise level.[97] In addition, the hydrophobicity of graphene caused clogging in the pore resulting from strong interactions between graphene and DNA nucleotides; however, hydrophilic modification of graphene with self-assembled monolayer of pyrene ethylene glycol has demonstrated to reduce the clogging.[131] 
Graphene-based nanopore research field is yet in its early stage and there are still many fundamental aspects to be investigated. The current limit of the graphene nanopore diameter needs to be even smaller so as to achieve the electrical sensitivity among the four different nucleotides of DNA molecule by ionic current measurements. It is also desired that translocation velocity of a DNA molecule through a graphene nanopore slows down below the current limit of 10-100 nucleotides/ $\mu$ sec.[101, 102] Together with graphene, other 2D crystals including $\mathrm{MoS}_{2}, \mathrm{BN}$, and heterogeneous layered materials have been extensively investigated to utilize their attractive properties for nanopore sensing. In section 5.2, we discuss a number of state-of-art research using 2D crystals for advancing the nanopore sensing technologies.

\subsection{Multi-electrode Array based Sensing Mechanism}

The MEA technologies have been developed especially in neuroscience for simultaneous intra- or extracellular recordings of hundreds of individual neurons. Electrodes record the action potential of a cell to monitor electrical activity of neuronal or cardiac cells (Fig. 3e,f).[15] These electrodes detect the signal of extracellular voltage signals generated by cells, when positioned outside the cell, which is referred to extracellular microelectrodes.[11] The extracellular microelectrode arrays (MEAs) allow the mapping of a neuronal network to physiological and pathological functions. Here we look into the MEAs technologies developed for extracellular recordings of neuronal or cardiac cells.[16] The MEAs technologies enable extracellular recordings at multi-sites over a long time to monitor signal transmission for in vivo or in vitro conditions. Simultaneous recording capability and the number/density of recording sites are dependent on the number of microelectrodes, and research in this field has aimed to increase the number density of the electrodes in a single MEA chip. To date, over 10,000 electrodes have been integrated in a single MEA chip for in vitro recordings by the state-of-art microfabrication technologies, [12, 18, 133, 134] whereas over a hundred of electrodes have been achieved for in vivo multi-electrodes.[135, 136] Current research aims to achieve simultaneous recordings and stimulations of hundreds of individual cells 
especially with long-term stable coupling of electrodes to neurons. Further challenge in this field includes recording extracellular signals with high signal-to-noise ratio in that the extracellular signals are typically hundred times smaller than intracellular signals.

\subsection{Optical Resonator-integrated Sensing Mechanism}

Graphene has been integrated with a surface plasmon resonance (SPR) based biosensor which detect biomolecular interactions on the sensing surface of graphene by the resultant local change in the refractive index near the surface. The alteration causes a shift in the resonance wavelength and the resonance shift is optically measured by attenuated total reflection (ATR) method.[19, 20, 22] Graphene improves the sensitivity of the SPR biosensor by enhanced adsorption of biomolecules on graphene surface, compared with metallic films such as gold.[23] In addition to the SPR-based technologies, patterned graphene micro/nano-ribbons was integrated with infrared spectroscopic techniques in order to use the unique electrical and optical property of graphene.[21]. Patterned graphene micro/nano-ribbons' properties of resonant absorption and tunability by electrostatic gating have allowed a high sensitivity, tunable plasmonic biosensor with label-free detection. We discuss various applications together with details in the applications section.

\subsection{Multi-functional Sensor Array based Sensing Mechanism}

Multifunctional sensing/stimulation system has been recently investigated, in comparison to a single functional component of sensing and stimulation systems. In research and clinical therapy of cardiology and neuroscience, multiparametric physiological sensing/recording and stimulation are important for high density mapping capabilities. The sensing/recording and stimulation components include temperature, $\mathrm{pH}$, and mechanical strain sensors, as well as optical stimulation and thermal/electrical actuators.[73, 75] This application has great potential for realizing non-invasive biomedical implantable devices and epidermal electronics providing quantitative measurement data for 
Review Article in Microelectronic Engineering

assessing disease and clinical monitoring.

\section{Fabrication Techniques for 2D Material based Bioelectronics}

Crucial steps to fabricate 2D material-based bioelectronic sensors, specifically graphene-based FETs, include patterning of the active graphene channel, fabricating source and drain electrodes, building fluidic systems for integration with reference electrodes, and overall functionalization. In this section, we discuss various techniques for each crucial step.

\subsection{Patterning of Transistor Channels}

After graphene is transferred onto the target substrate, graphene is patterned to create a transistor channel for individual, isolated FETs. Patterning techniques are categorized into conventional photolithography,[137-141] direct patterning using electron beam (e-beam),[142-147] ion beam,[148] lasers,[149, 150] and direct growth of patterned graphene.[64, 151-155] Conventional photolithography uses patterned photoresist to selectively passivate graphene. Subsequently, unprotected areas of graphene are chemically etched by exposure in plasma etching.[82] This method can create a graphene channel with width down to $\sim 20 \mathrm{~nm}$ with edge roughness of a few nanometers. However, it requires a number of process steps and results in degradation in electronic transport properties due to residual photoresist and contaminants, which are inevitably introduced in transfer processing of graphene on a desired substrate. Moreover, it poses potential compatibility issues of organic solvents used in photolithography with polymeric substrates, which are often used for emerging applications of flexible and wearable electronics.

Direct patterning method uses aforementioned energetic sculpting tools to create patterns on graphene. Feature size and roughness of edges yielded by this method are constrained by instrumentation. Specifically, the feature size yielded by e-beam lithography is primarily dependent on the e-beam size as well as on scattering and propagation of electrons in a resist material. Currently, state-of-art e-beam tools can achieve $10 \mathrm{~nm}$ line-width.[156] A direct patterning technique based on laser scribing was shown to 
pattern sophisticated design in a single step process with sub-20nm feature size and enhanced conductivity of patterned graphene.[149] This technique does not require masks, templates, nor postprocessing. Moreover, it allows tunability of the electronic properties by varying laser intensity. However, the process is limited to serial processing and has limited scalability. Although laser ablation based direct patterning allows nanometer-scale patterning, it requires an expensive ultrashort pulse laser with a femtosecond range.[150]

Direct growth is a bottom-up synthesis approach, allowing scalable patterning.[64, 151-155] This method can grow large-scale graphene films on a pre-patterned catalytic substrate. Subsequently, grown graphene films are lifted off from the catalytic substrate through etching and transferred to a desired substrate. Based on this approach, various patterns and widths at atomic precision were demonstrated by using precursor monomers deposited onto a catalytic substrate.[155] Although the direct growth method allows scalable patterning, the catalyst nonetheless still needs to be patterned by photolithography prior to the growth step.

\subsection{Fabrication of Source/Drain and Gate Electrodes}

An essential step following the patterning step is making source and drain electrodes.[132, 157] Metal contacts are thermally deposited directly onto the graphene with a shadow mask,[158-160] or contact patterns are created on a substrate by photolithography [161] or e-beam lithography [87] and subsequently metal contacts are thermally deposited on top.[162]

The metal-graphene contact is a potential limiting factor of high performance graphene FET devices, and the preparation of a truly Ohmic contact between metal electrodes and 2D materials are not trivial. Adhesion issues on the graphene-metal contact, primarily influenced by the adhesion properties of graphene, result in delamination of metal electrodes and increase in contact resistance.[163, 164] To form high quality Ohmic contact with intimate contact between a metal and graphene and reduce the contact resistance, proper electrode metal schemes need to be selected. Metal contacts commonly used include a 
transition metal (e.g. Ti, Pt, or Pd) as an intermediate layer and an Au film.[3]

For the use of FET devices in biological applications, a stable passivation of the source and drain electrodes against electrolyte solutions is required to block interaction with aqueous solution and avoid electrolysis in addition to unwanted leakage current. Common materials used for passivation include dielectric materials[162] and polymers.[83]

\subsection{Integration of Reference Electrodes}

Electrolyte-gated FETs (EGFET) are widely used in biological sensing. EGFET requires the use of large external gate electrode, for example a separate silver/sliver chloride reference electrode or a metallic wire of gold, silver, or platinum. $[3,165]$ Devices developed for research often use temporary electrical connections to source and drain electrodes, and a reference electrode in open reservoir of an isolated well filled with electrolyte submerging the graphene channel. For practical graphene-based FET biosensors, Stine et al.[162] developed a microfluidic measurement system with a flow cell and integrated electrical connections to source, drain and reference electrodes. This measurement system can be switched rapidly between FET biosensing devices.

A fabrication technique to enable scalable integration of reference electrodes was demonstrated. Vieira et al.[166] developed a planar architecture for graphene EGFET in which source, drain, and gate are in the same plane. This planar architecture with integrated in-plane gates allowed for $200 \mathrm{~mm}$ waferscale fabrication of graphene EGFET array devices (280 FETs). This architecture also enabled miniaturization of array devices by a compact, integrated design. The device miniaturization allows for portability and ease of use and has potential for applications in portable, disposable point-of-care medical devices. Moreover, this architecture allows for device fabrication using conventional photolithography, which has the potential for low-cost mass production of biosensor devices.

\subsection{Functionalization}


A crucial step in building FET biosensors is endowing graphene with biological specificity by attaching proper recognition elements as pristine graphene shows inertness to many chemical reactions. Covalent attachment of recognition elements to graphene necessarily perturbs graphene's $\mathrm{sp}^{2}$ hybridization to $\mathrm{sp}^{3}$ hybridization, and alters electronic properties of graphene leading to reduction of carrier mobility.[3, 37, 167] Noncovalent attachment of probe molecules with a two-step functionalization procedure has been widely used in graphene FET biosensors because it does not perturb the electronic structure. First, aromatic molecules such as 1-pyrene butanoic acid, which contain a function group,[87, 168] are physically adsorbed while aligned with graphene lattice through $\pi$ - $\pi$ stacking.[169] Next, biomolecular probes are attached to the functional group and stably attached to the graphene surface without disturbing the electronic structure. In another non-covalent method, nanoparticles, for example gold nanoparticles, are physically adsorbed on the graphene surface to attach probe biomolecules.[126] Gold nanoparticles are subsequently functionalized with a thiol group to form anchoring sites where probe biomolecules are attached. In addition to functionalization step, passivation of the graphene surface is required to avoid non-specific adsorption, while preserving selectivity and probe-target affinity. A blocking buffer with a combination of Tween 20 and bovine serum albumin (BSA) is usually used for the surface passivation.[126, 170]

\section{Applications}

The previous section introduced transduction and detection technologies with their physical mechanisms and approaches for integrating multifunctional sensing. In this section, we review various applications that have been recently developed based on the mechanisms and approaches introduced in Section 3. Applications introduced in this section are categorized into three key topics: (1) chemical/molecular sensors, (2) nanopore-based sensors and (3) neurobiological and cardiac sensors.

\subsection{Chemical and Molecular Sensors}


Biosensors [24-26, 42] comprise two main components; (1) biological sensing element such as enzymes, antibodies, and nucleic acids, which is coupled to a transduction element for detecting an analyte through binding interactions and (2) transduction element, which transforms the signal resulting from the interactions to a quantifiable and measureable signal such as electrical current. We mainly discuss graphene-based biosensors,[87, 120] but also discuss other emerging 2D materials, for example, $\mathrm{MoS}_{2 \cdot[51]}$

\subsubsection{Molecular Sensors based on Amperometric/Conductometric Measurements}

Among various sensing targets, glucose and cholesterol biosensors are especially emphasized in that such target molecules are clinically important for diagnosing and preventing common diseases such as diabetes and cardiac disorders. Glucose detection is based on the current response of graphene and its derivatives arising from enzymatic reaction, specifically the oxidation of hydrogen peroxide $\left(\mathrm{H}_{2} \mathrm{O}_{2}\right)$ produced during the enzymatic reaction of glucose. An enzymatic electrode based on graphene derivatives such as graphene oxide (GO) was obtained by covalently attaching glucose oxidase (GluOx) to the GO sheets.[171] The glucose oxidase-immobilized graphene oxide (GluOx-GO) enzyme electrode showed high sensitivity $\left(8.045 \mu \mathrm{A} \cdot \mathrm{mM}^{-1} \cdot \mathrm{cm}^{-2}\right)$, a wide linear range up to $28 \mathrm{mM} \cdot \mathrm{mm}^{-2}$, good reproducibility, and storage/operational stability. Notably, biocompatibility of the GO film with human epithelium cells was demonstrated as a practical biosensor device for clinical diagnosis. Besides the GO, graphene was assembled with GluOx to achieve even higher sensitivity $\left(\sim 110 \mu \mathrm{A} \cdot \mathrm{mM}^{-1} \cdot \mathrm{cm}^{-2}\right)$ together with a low detection limit $(\sim 10 \mu \mathrm{M})$.[172, 173] A novel nanocomposite [174] of graphene, gold nanoparticles (AuNPs), GluOx, and Nafion were also developed, showing a lower detection limit of $\sim 5$ $\mu \mathrm{M}$, compared with the aforementioned electrodes. The AuNPs were used for immobilizing biomolecules as well as enhancing the signal transduction by promoting electron exchange at the protein/electrode interface.[39] Nafion, a perfluorinated sulfonated copolymer, served thermal stability, biocompatibility, and mechanical strength to support the electrode. The GluOx-GO and GluOx-graphene electrodes 
demonstrated a promise of graphene and its derivatives for potential utilities in a bioelectroanalytic method using their electrochemical features and immobilized enzymes.

A reduced graphene oxide (rGO)-based micro-electrode array was also demonstrated for the detection of hydrogen peroxide $\left(\mathrm{H}_{2} \mathrm{O}_{2}\right)$ with a lower detection limit and a larger current signal, as compared with macroscopic large patterns of a rGO electrode.[175] Moreover, rGO-based microelectrode array was fabricated on a flexible substrate by nanoimprint lithography for scalable fabrication of a highly sensitive and flexible biosensing platform.[14]

For amperometric cholesterol biosensors, [8] $\mathrm{H}_{2} \mathrm{O}_{2}$ is produced from enzymatic reaction between cholesterol and cholesterol oxidase (ChOx). Graphene-based electrodes were used for the detection of $\mathrm{H}_{2} \mathrm{O}_{2}$ by the electrochemical sensing similar as the glucose detection mechanism. Graphene was developed into nanocomposite electrodes assembled with polyvinylpyrrolidone (PVP) and polyaniline (PANI), graphene/PVP/PANI electrodes.[176] The nanocomposite electrodes enhanced sensitivity for cholesterol concentration with an amperometric method, attributable to increase in electrochemical conductivity. Graphene tends to agglomerate owing to van der Waals attraction when dispersed in solution, but PVP improved the dispersity as a stabilizer. The PANI, conducting polymer, was used to further enhance the sensitivity, benefiting from its excellent electrochemical properties, high stability, low toxicity, and easy functionalization. The developed system showed a low detection limit of $1 \mu \mathrm{M}$ for cholesterol together with a wide linear range $(50 \mu \mathrm{M}-10 \mathrm{mM})$. Moreover, a paper-based biosensor was fabricated by electrospraying, a simple process to form the nanocomposite electrodes, showing potential for low cost medical diagnostic devices and disposable biosensors.[176] The 2D materials including graphene and $\mathrm{MoS}_{2}$ provide various advantages benefiting from its 2D layered structure including better binding efficiency, high analyte selectivity, and small loading capacity compared with other peroxidase like catalytic activity materials such as nanoparticles.

\subsubsection{Field-effect Transistor based Sensors}


Review Article in Microelectronic Engineering

A $\mathrm{MoS}_{2}$-based FET biosensor showed great promise for single molecule detection with extremely high sensitivity of $\sim 100$ femtomolar concentration of streptavidin and Immunoglobulin G (IgG) that interact with biotin absorbed on a $\mathrm{MoS}_{2}$ substrate (Fig. 4a).[51] The effect of the number of layers on the device performance was studied and it was found that few-layer $\mathrm{MoS}_{2}$ was more beneficial to device performance compared to mono-layer $\mathrm{MoS}_{2}$. Moreover, $\mathrm{MoS}_{2}$ enabled exceptionally high sensitivity together with easy device fabrication based on patterning due to the 2D layered structure. Most importantly, this $\mathrm{MoS}_{2}$-based FET biosensor showed the potential of other 2D materials, $\mathrm{MoS}_{2}$, other than graphene for next generation of a highly flexible and low-cost biosensor device.

FET arrays comprise of a multitude of functional units of single FET. Graphene-based FET device array has also shown its potential for electroanalytical applications.[14, 177, 178] An FET DNA sensor array using CVD graphene as electrophoretic electrodes and sensing FET has shown the detection of target DNA with the sensitivity of $1 \mathrm{pM}$ while using the graphene electrophoretic electrodes for active site-specific immobilization of probe DNA (Fig. 4b).[178]

For the detection of gas or vapor molecules, relatively low sensitivity and slow response time have been challenging for graphene-based FET sensors as well as existing nanoelectronic gas sensors. In this regard, a graphene-based FET sensor with a unique detection mechanism was reported by Kulkarni et al.[179] The detection of various vapor analytes including pentane, hexane, benzene, chlorobenzene, and so on was demonstrated by using a mechanism based on modulated conductance in a graphene channel. The modulation of the conductance was induced by the oscillating molecular dipole of a vapor molecule resulting from alternating current (AC) excitation. Such novel mechanism enabled rapid ( 0.1 s) and sensitive ( 1 parts per billion, ppb) detection of various vapor analytes, compared with the sensitivity (10s of ppb) and response time (>100s) of nanoelectronic devices using nanomaterials such as carbon nanotubes (CNTs), graphene and TMDs.

Biomimetic sensors such as artificial nose and tongue based on graphene-based FET have also been conceived. An olfactory sensing device was developed into a flexible and transparent FET platform 
(Fig. 4c-e).[88] Bilayer graphene used as a sensing material was functionalized with a human olfactory receptor for the detection of a particular odorant. Most importantly, the bioelectric nose (B-nose) showed ultrahigh sensitivity with a low detection limit of $\sim 0.04$ femtomolar (fM). The functionalized graphene enabled exceptionally high sensitivity, attributable mainly to high carrier mobility of graphene. Long-term stability and excellent mechanical flexibility were also demonstrated. This device has potential for a broad range of applications including environmental monitoring, food safety, and disease diagnostics with the capability of recognizing various odorants. Moreover, the flexible and transparent platform will enable a wide range of applications.

\subsubsection{D Material-integrated Optical Sensors}

2D materials such as graphene [19] were also used to enhance the performance of surface plasmon resonance (SPR) based optical biosensors by a hybrid approach (Fig. 4f,g).[37] A hybrid system of silicon nanowire (SiNW) nanostructure arrays functionalized with monolayer graphene supported highly localized optical resonances. The hybrid system enabled high sensitivity, label-free and low-cost biosensing based on the detection of surface adsorption events of a target analyte. The detection limit of $\sim 300 \mathrm{pM}$ protein $\mathrm{A} / \mathrm{G}$ binding with $\mathrm{IgG}$ was shown with a resonance wavelength shift of $5.4 \mathrm{~nm}$. The resonance wavelength shift was led by the change in effective refractive index of the hybrid structure that supports certain resonance mode (or wavelength). Importantly, graphene served as a functionalization layer to adsorb probing biomolecules, such as IgGs for detecting protein A/G. This sensing platform showed great potential for investigating protein-protein interactions and developing on-chip silicon-based photonic biosensors. Moreover, this hybrid approach can be used to develop biosensors with a variety of semiconductor materials as well as various geometries.

It was also shown that graphene can enhance the SPR sensitivity by three-fold with a hybrid substrate that integrated graphene with a silver film, compared with conventional gold-film based SPR biosensors.[180] Moreover the detection was based on SPR imaging, which has potential for high 
throughput and multiplexing sensing of biomolecular interactions.

In addition to the hybrid approach, patterned graphene was developed for a sensing element that supports SPR in mid-IR wavelengths.[21] A tunable plasmonic biosensor was developed based on unique electro-optical properties of graphene including broadband absorption and electrostatic tuning of carrier density. Various proteins were shown to be selectively detected at different frequencies in mid-infrared with dynamically tuned plasmonic resonance in a graphene nanoribbon array. The dynamic tuning of the plasmonic resonance is a key advantage over traditional sensing materials such as gold. More notably, the tunable plasmonic resonance enabled broad spectral sensing with a single biosensor device. The plasmonic biosensing with the graphene nanoribbon array provided superior sensitivity with a large spectral shift led by stronger field confinement of graphene using the nanoribbon pattern. The stronger field confinement also enhanced light-protein interactions, compared with metallic plasmonic sensors. Most importantly, this demonstrates great prospects for a next generation graphene-based plasmonic biosensor with enhanced sensitivity and tunable spectral selectivity.

\subsection{DNA Sequencing Technology based on 2D Material Nanopores}

Nanopore fabrication in suspended multilayer graphene sheets by electron beam ablation lithography was firstly reported by Fischbein and Drndic in 2008.[181] This has led to studies of DNA translocation through graphene nanopores.[7, 97, 131, 182] In 2010, translocation of double-stranded DNA molecules through nanopores in suspended graphene membranes was demonstrated by several research groups; Either mechanically exfoliated graphene [131] or CVD grown graphene [7, 97] were used in their experiments (Fig. 5a). The diameter of nanopores used in the experiments was $\sim 10 \mathrm{~nm}$. As the translocation speed has been too fast to resolve individual nucleotides by ionic current in all the experiments, it remains a challenge to achieve single base resolution. In the regard, a suspended graphene nanoribbon on a fluidic nanochannel has shown the approach of controlling the DNA translocation speed.[183] DNA strands translocated steadily with controlled speed through a fluidic nanochannel 
underneath the graphene nanoribbon by an externally applied electric field. This approach was based on the measurement of the conductance changes of the graphene nanoribbon led by $\pi$ - $\pi$ interactions between nucleobases and the graphene nanoribbon.

Graphene nanopore-based sensing stimulated other 2D materials such as BN,[95] and monolayer $\mathrm{MoS}_{2}$ (Fig. 5b) [94] for nanopores sensing. Monolayer $\mathrm{MoS}_{2}$ is an emerging material owing to its semiconducting properties with a direct band gap in contrast with gapless graphene. Nanopores with varying sizes $(D=2-20 \mathrm{~nm})$ has been fabricated on monolayer $\mathrm{MoS}_{2}$ by using a transmission electron microscope (TEM). Translocation of double-stranded DNA with a better signal-to-noise ratio (>10) has been shown experimentally, compared to $\mathrm{SiN}_{\mathrm{x}}$ based nanopores. Notably, while graphene requires hydrophilic treatment to avoid clogging resulting from strong interaction between graphene and DNA nucleotides, $\mathrm{MoS}_{2}$ monolayer requires no additional surface treatment. Recently, an electrochemical method controllably fabricating nanopores in monolayer $\mathrm{MoS}_{2}$ was presented by Feng et al.[91, 184] Such method is convenient and scalable with subnanometer precision as compared to conventional nanopore fabrication method using TEM. Moreover, a novel nanopore fabrication technique by heating a gold nanorod with a laser was reported (Fig. 5c,d).[98] This technique has the potential for parallel nanopores fabrication together with tunable nanopores dimensions. In comparison, the traditional sequential method using an electron beam is considered to be time-consuming, expensive, and nonscalable, and difficult to control for subnanometer pore size.

In addition to graphene and $\mathrm{MoS}_{2}, \mathrm{BN}$ is also attractive for nanopore sensing technology due to complementary properties to graphene such as high mechanical strength and durability, high corrosion resistance, high dielectric property and a large band gap. The thin structure $(\sim 1.1 \mathrm{~nm})$ is comparable to the spacing between bases of single-stranded DNA $(0.32-0.52 \mathrm{~nm})$ and can possibly enable single-base resolution. The insulating property of $\mathrm{BN}$ in high ionic concentration is superior to graphene, leading to a higher signal-to-noise ratio. Liu et al. reported first electronic measurement of DNA translocation through BN nanopores.[95] For a nanopore sensing technology, there are challenges to resolve, including slowing 
down the translocation speed and achieving single-base resolution.

\subsection{Neurobiological and Cardiac Sensors}

In this section, we review the state-of-the-art neural micro-electrode array technology based on 2D materials. In addition, multifunctional bioelectronic sensor array technology for non-invasive, longterm recordings of cardiac signals is also discussed. The multifunctional biosensor array technology has similar requirements as the micro-electrode array technology, which has been developed for high spatial density, simultaneous, neurophysiological/electrophysiological recording. The multifunctional biosensor array technology and the micro-electrode array technology have potential as an implantable device that requires reliability for long-term monitoring of physiological signals and biocompatibility together with mechanical flexibility and compliance in contact with soft tissues. The micro-electrode array device is often integrated with other elements for optical imaging/stimulation as well as optogenetic modulation of tissues. Integration of complementary functions (i.e., electrical and optical) will be also discussed in this section.

\subsubsection{Multi-electrode Arrays}

We focus on graphene as an electrode material in this section.[15, 106, 178] Neural interfacing devices have potential for neuroscience research as well as clinical therapy of neurological disorders such as Parkinson's disease, stroke, and spinal injury.[111] Recently, optogenetics has emerged as a new technique modifying neural cells to be responsive to optical stimulation.[185, 186] The neural interfacing device of micro-electrode arrays has been desired to integrate with the optogenetic stimulation system. Graphene has unique combination of optical and electrical properties for this application, such as excellent electrical conductivity, broadband transparency, and mechanical flexibility/strength. Conventional micro-electrode arrays have used indium-tin oxide (ITO). However, ITO material is not transparent in ultraviolet and infrared, and is mechanically brittle. Park et al. introduced a graphene-based 
micro-electrode array device for neural interfacing, and demonstrated optogenetic stimulation of cortical tissues and optical in vivo imaging of cortical vasculature together with electrophysiology (Fig. 6a,b).[15] Most notably, graphene was used as a transparent electrode material because of broad spectral transparency from ultraviolet to infrared with a low absorption of $\sim 2 \%$ in the visible range. Du et al. demonstrated long term stability for detection of neural activities by using fabricated graphene MEAs

(Fig. 6c,d).[106] Graphene enabled high electrical conductance, optical transparency, and biocompatibility for the device, compared with traditional electrode materials including titanium nitride, titanium, and ITO.

\subsubsection{Field-effect Transistors}

Graphene-based FET arrays have shown the detection of extracellular signals of action potentials from cardiomyocyte cells.[187] Most notably, the propagation of action potentials across the cells was monitored with graphene-based solution-gated FET (SGFET) arrays and the propagation speed was determined to be $12-28 \mu \mathrm{m} / \mathrm{ms}$. The graphene SGFET enabled high signal-to-noise ratio, compared to Sibased devices. This study showed the potential of a graphene FET array technology for investigations in cardiology, together with easy integration of such technology on flexible substrates by using CVDgraphene. Cohen-Karni et al. also demonstrated a graphene SGFET device that carried out recording of extracellular signals from beating cardiomyocyte cells.[188] The recording was based on a robust interface between graphene and the cells.[189] Notably, simultaneous recording of extracellular signals from the same cell with different types of devices, graphene FET and silicon nanowire FET devices, provided the comparison of temporal resolution of the recordings between the devices. It was discovered in the analysis of the conductance versus time data that the temporal resolution of the two types of device were similar to each other. Although the potential of graphene FET arrays technology was demonstrated by these studies, the integration of graphene FET arrays on flexible substrates is desired, together with long-term robust interfacing for developing multi-functional sensing devices for cardiac research and 
clinical therapy. Such multi-functional sensing devices are discussed in the following section.

\subsubsection{Multi-functional Sensors}

For cardiac research and clinical therapy, tools for high-density sensing/mapping of multiparametric physiological signals and stimulation have long been desired. In addition, conformal devices have been needed to retain reliable interfacing between cardiac tissues and sensing components for implantable and chronic uses. Recently, flexible electronics has emerged as a technology to integrate multifunctional sensors, electronic and optoelectronic elements on three dimensional elastic membranes (Fig. 7a,b).[73] The 2D membrane was shown to make conformal contact with the epicardial surface of a heart for reliable sensing/mapping. The integrated components included inorganic light-emitting diodes for optical mapping, silicon ( $\mathrm{Si}$ ) nanomembranes for strain gauges, gold electrodes for electrical sensing and stimulation, iridium oxide ( $\mathrm{IrOx}) \mathrm{pads}$ for $\mathrm{pH}$ sensors, and $\mathrm{Au}$ serpentine resistors for temperature sensors/heaters. This device has demonstrated a great potential in interrogating extensive information on cardiac activities for clinical diagnosis and therapy.

The approach of integrating multifunctional sensors on a thin flexible substrate was also applied to develop a stretchable, conformal, multifunctional electronic sensor devce $[73,75,107]$ for clinical monitoring of skin diseases and surgical wound healing (Fig. 7c-e).[75] Micro metal arrays of resistors precisely demonstrated the measurements of skin temperature and thermal conductivity. Notably, this biocompatible and non-invasive epidermal electronic device shows potential to monitor skin physiology for providing quantitative assessment.

\section{Remarks and Outlook}

In this paper, we have reviewed various bioelectronic devices and applications using 2D materials. We have discussed the unique properties of the $2 \mathrm{D}$ materials that enable such highly sensitive, flexible, and transparent systems. Notably, graphene and its related materials enable biocompatibility and long- 
term viability required for biomedical and clinical uses. The flexible, thin, and conformal platform based on 2D nanomembrane materials has been highlighted for broad potential in recently emerging areas, for example, epidermal/wearable electronics and stretchable/flexible bioelectronics. Together with detection mechanisms and advanced technologies of array platform and multifunctional sensor systems, various applications have been introduced, including chemical/molecular sensors, nanopore-based sensors, and neurobiological and cardiac sensors. In this section, we discuss ongoing efforts and promising approaches to advance the device performance for mechanically flexible/stretchable and conformal systems as well as higher sensitivity. We introduce the following approaches: (1) strain engineering for modulating and enhancing properties of the 2D materials, (2) transformation of intrinsic 2D structures to 3D topographical structures for better performance and tissue-probe interfaces, (3) novel hybrid approaches of integrating different nanomaterials and (4) biodegradable electronics.

\subsection{Strain Engineering of 2D Materials}

Altering the electronic structure of $2 \mathrm{D}$ materials such as graphene and TMDs is possible by applying mechanical strain. The tuning of the electronic structure changes their electronic and photonic properties. Modifying their properties allows the utilization of resultant behavior for promising flexible electronic/optoelectronic applications. Techniques for deterministically applying strain have been investigated extensively [80,177, 190,191], for example stretching or bending of a supporting elastic substrate with transferred graphene and other 2D materials. Thermal expansion and contraction of a substrate also showed controlled crumpling to induce strain uniformly over large area (Fig. 8a,b).[192] The strained graphene has much potential particularly for transistor devices because of the possibility of introducing a band gap into the gapless material.

Furthermore, through the application of strain, chemical reactivity of graphene can be selectively enhanced.[190] It has been found that the reactivity of graphene depends on the internal strain on its

carbon $\mathrm{sp}^{2}$ bonds, because charges localized around the strained carbon bonds increase binding energy for 
molecules and enhances adsorption for nanoparticles.[193]

Such wrinkling Wrinkling/crumpling approach can also be used in plasmonic biosensing to achieve high sensitivity. Three-dimensional hybrid nanoplasmonic structures were introduced by the crumpling of a graphene film decorated with gold nanoparticles (AuNPs) (Fig. 8c,d).[194] Notably, the crumpled graphene-AuNPs hybrid nanostructure demonstrated an order-of-magnitude higher surface enhanced Raman spectroscopy (SERS) signal than an identical but otherwise flat graphene-AuNPs substrate. The strain engineering of graphene and other 2D materials is an emerging field of research, and further investigations and developments is still needed to realize the full potential.[177]

\subsection{D Topographic Engineering of 2D Materials}

Structured 2D materials by mechanical strain, such as wrinkled graphene, can enhance performance by the modulated electronic [188, 195-198] and optical properties.[199, 200] Moreover, strained 2D materials has been employed to impart larger stretchability and high flexibility with the 3D topography of formed wrinkled structures to beyond the intrinsic strain limit, for example, $\sim 25 \%$ for graphene.[190] This enhanced stretching capability will benefit applications in flexible and epidermal electronics together with enhanced performance.[53, 54]

For implantable biomedical sensors and diagnostic tools, the capability to measure physiological signals is important. The signals are measured by reliable contact between the device and biological tissue. Three dimensional topography of wrinkled structure can be used to make long-term reliable interface between sensing probe and tissues/cells. This approach can be applied to make MEA devices for sensing electrophysiological signals from skin to the surface of heart and brain. Recently, the wrinkled structure has been employed to develop into a stress-absorbing MEA device (Fig. 8e,f).[80] Attached to the surface of a rat heart, the device showed reliable mechanical contact while measuring electrophysiological signals under $\sim 110 \%$ compressive strain. In addition to the approach using a highly flexible, wrinkled, thin polymeric film, the approach integrating graphene onto deterministically patterned 3D microstructured 
surfaces was introduced to show the potential for developing devices that provide long-term conformal adhesion between devices and human body.[201] The graphene-integrated microstructured surfaces can be developed to be 3D MEAs for measuring electrophysiological signals from human body or cells/tissues. In summary, adhesion between probe and tissue can be enhanced by engineering 3D topology of 2D materials, and the enhanced contact can provide reliable in vivo monitoring of biological signals. This technology will find useful applications in wearable electronics and implantable biomedical devices.

\subsection{Hybrid Systems of Low-dimensional Materials and 2D Materials}

For bioelectronic sensor applications, hybrid systems integrating other low dimensional materials with 2D materials [69] can be used as a sensing electrode material. Biomolecules-nanoparticle hybrid systems were developed for photoelectrochemical sensors and optoelectronic systems.[202] The applications include functionalized gold nanoparticle for the DNA analyte to use AuNPs as a carrier for a probing biomolecule of a nucleic acid. The coupling between nanoparticles and biomolecules enhances electrical communication between a probing molecule and electrode. This approach can be applied to advance the FET-based electrochemical biosensors. Such hybrid electrodes of biomolecules-NPs-2D material may enhance electrical properties of the electrode by increasing interfacial area and enhancing the electrical communications formerly made between an electrode of only 2D materials and probing molecules. Besides gold nanoparticles, semiconductor quantum dots can also be integrated with 2D materials electrodes to utilize the coupling of light and electrical energy to the hybrid systems for photoelectrochemical and plasmonic biosensors.[194]

Recently, vertical heterostructures made by stacking different 2D materials such as graphenehBN have been extensively investigated.[28] Notably, the heterostructures can complement graphene's drawback such as the absence of a bandgap by modifying electrical properties of graphene as a result of the basal interactions.[92] Merging advantageous properties together by employing heterostructures may 
have important applications in optoelectronic biosensors and bioelectronic sensors. Yet, heterostructures have been a recent area of active research and methods to efficiently and chirally assemble different $2 \mathrm{D}$ crystals are still being investigated.

\subsection{Biodegradable Electronics}

Bioelectronic systems have been desired to be made of transient biodegradable materials and nontoxic materials (Fig. 8g,h).[72] For implantable biomedical applications, such systems can have direct impact in that biodegradable systems can minimize surgical procedure by making it dissolvable in human body. Biocompatible and biodegradable electronic systems will have potential in applications ranging from resorbable medical devices and environmental monitors to disposable electronics.

\subsection{Challenges on 2D Materials based Bioelectronics}

The limitation of biosensing with graphene is its inertness to chemical reaction for functionalization [167] and hydrophobic nature of graphene surface. The hydrophobicity of graphene surface [203] in ambient environment leads to nonspecific adsorption of undesirable molecules,[204] which poses a challenge for specificity of detection. As a result, graphene needs to be functionalized with recognition molecules for detection of target molecules. Approaches to functionalizing graphene include covalent and noncovalent methods. Covalent method allows for effective, stable, specific functionalization, but it perturbs the intrinsic electronic structure by converting the carbon bonding from $\mathrm{sp}^{2}$ to $\mathrm{sp}^{3}$. As a result, it inevitably alters physical properties of graphene, for example, yielding substantial reduction in carrier mobility. On the contrary, noncovalent method is based on van der Waals or $\pi-\pi$ interaction which facilitates physical adsorption. While various molecules can be immobilized on graphene through physical adsorption, it could also lead to the non-specific adhesion of undesirable molecules, and passivation molecules must be used to avoid non-specific adhesion on non-functional sites. Consequently, to enable specific detection for graphene biosensors, reliable and non-intrusive 
functionalization procedures need to be developed to preserve intrinsically excellent materials properties of graphene and while maintaining high performance in sensing. In addition, further research is required for better understanding of interactions of chemicals and biomolecules at the graphene surface, i.e., how such interactions affect electronic transport properties of graphene, the adsorption mechanism, orientation of biomolecules on graphene, as well as physical and chemical properties of the intrinsic graphene surface. Such understanding may lead to advancement of various applications of graphene FET biosensors.

For FET sensor applications based on 2D materials, low-frequency noise is a crucial limitation on the performance of sensing.[3] This noise is commonly referred to $1 / f$ noise or flicker noise, and ranges in frequency of less than a few kilohertz.[205] Furthermore, downscaling to nanometer-scale devices is desired to develop compact, portable biomedical device applications with reduced power consumption. However, this scaling down can further increase $1 / f$ noise, and hence low-frequency noise is of a significant consideration in development of practical applications, especially for high-throughput array devices requiring fabrication of nanometer-scale devices. The investigation of $1 / f$ noise is yet in an early stage to explain specific aspects in 2D materials.[206] A comprehensive theoretical model needs to be developed, and the influence of gate-bias-voltage on low-frequency noise characteristics and the influence of metal contacts and binding of analyte molecules onto the surface of a $2 \mathrm{D}$ material channel on $1 / f$ noise have to be further investigated.

2D semiconductor materials such as TMD monolayers having a band gap have recently emerged as promising channel materials. Specifically, a $\mathrm{MoS}_{2}$ FET biosensor demonstrated high sensitivity together with excellent electrostatic gating when it is operated in the subthreshold region enabled by the presence of a bandgap.[51] Moreover, $\mathrm{MoS}_{2}$ allows for downscaling of device dimension (i.e. decreasing the channel length) with increase in sensitivity for detection of low concentrations of small biomolecules such as DNA or small proteins $(<10 \mathrm{~nm})$. The device scalability also allows for reduction of power consumption as well as high density architectures for sophisticated multiplexed biosensors. Importantly, large-scale, uniform-quality $\mathrm{MoS}_{2}$ atomic layers are required for large-scale device fabrication of 
multiplexed biosensors and low cost mass production of biosensor devices. However, currently the TMD monolayer synthesis technique is yet in an early stage of development for a large-area, uniform $\mathrm{MoS}_{2}$ monolayer material. The recent development of large-scale uniform $\mathrm{MoS}_{2}$ synthesis technique [207] can allow for low-cost mass production as well as fabrication of multiplexed biosensors. A low-temperature direct growth technique of large-scale $\mathrm{MoS}_{2}$ on flexible substrates needs to be developed to achieve costeffective flexible biosensor platforms.

\subsection{Future Directions of 2D Materials based Bioelectronics}

Since the first report of graphene FET,[2] significant progress has been achieved in areas including large-scale, high-quality, low-cost 2D material synthesis technology, development of methods for the detection, identification, and quantification of biomolecules, and high-throughput real time measurement of cellular behavior. The broad range of examples described in our review summarizes such achievements. However, current methods for building micro-fabricated bioelectronic devices are not well established for practical applications and commercialization of 2D material-based bioelectronics. Academic research fabrication facilities or small manufacturers have supplied micro-fabricated biomedical devices for proof-of-concepts, including integrated FET biosensors, multielectrode arrays, etc. Yet, conventional fabrication approaches for semiconductor technologies need to be applied to fabrication and processing of sophisticated 2D material-based biosensors for low cost mass production. For practical applications, biocompatible, durable, and reliable device packaging remains a challenge, and reliable integration with microfluidic systems such as microfluidic purification modules for biomolecule detection from biofluids has to be developed in consideration of potential problems by liquid interfacing. In addition, among many applications of 2D material-based bioelectronics, one of most impactful areas could be wearable and implantable biomedical sensor applications, which requires a flexible platform of 2D material-based biosensors. The development of a flexible and stretchable platform based on 2D materials will be necessary. Finally, device and material's biocompatibility as well as new technologies 
Review Article in Microelectronic Engineering

and approaches for harvesting and storing energy to power wearable and implantable devices need to be investigated.

\section{Acknowledgements}

This work was supported by the Air Force Office of Scientific Research/Asian Office of Aerospace Research Development (AFOSR/AOARD) Nano Bio Info Technology (NBIT) Phase III Program (AOARD-13-4125), the Brain Research Foundation Fay/Frank Seed Grant (BRFSG-2014-10), American Chemical Society Petroleum Research Fund (53270-DNI10), and the National Science Foundation (NSF) CAREER Award 1554019. 


\section{Figure Legend}

Figure 1. Bioelectronics with 2D materials and 2D nanomembrane materials. (a) Components of a biosensor for the detection of target samples with receptors. Reprinted from [58] with permission from Molecular Diversity Preservation International (MDPI). (b) Illustration of 2D atomically-thin materials with various atomic structures, including graphene, h-BN and TMDs. Reprinted with permission from [53], copyright 2010, Nature Publishing Group (NPG). (c) Schematic illustration of graphene synthesis on a copper substrate by CVD: copper foil with native copper oxide (left), the nucleation of graphene islands on the copper foil in $\mathrm{CH}_{4} / \mathrm{H}_{2}$ at $1000{ }^{\circ} \mathrm{C}$ (middle), development of polycrystalline graphene domains (right). Reproduced from [67] with permission from The Royal Society of Chemistry (RSC).

Figure 2. Unique 2D materials' properties for bioelectronics. (a) Bright-field images of human neural stem cells grown on glass (left) and graphene (right) after one month. Note the larger number of the cells on graphene than on glass. Reprinted from [77] with permission of John Wiley \& Sons, Inc. (b) Optical image of a deformed complementary metal-oxide-semiconductor (CMOS inverter array. The inset SEM images on lower left side show various deformation modes in diagonal stretching, twisting, and bending, respectively. Reprinted from [79], Copyright @ 2008 National Academy of Sciences.

Figure 3. Detection and sensing mechanisms of various bioelectronic systems based on FETs, nanopores, and MEAs. (a) Schematic drawing of graphene-based FET biosensors for detection of bacteria. The inset SEM image shows an E. coli on antibody-functionalized graphene. (b) Electrical characteristic curves of antibody functionalized graphene FET before and after binding of E. coli.. (a, b) Reproduced from [120] with permission, Copyright (c) 2011 The RSC. (c) Schematic illustration of graphene nanopore devices with a 1-5 nm-thick few-layer graphene suspended over a hole on a silicon 
nitride membrane. A bias voltage (VB) is applied between reservoirs to drive DNA through the nanopore. (d) Measured current over time during DNA translocation through graphene nanopore. Reprinted with permission from [97], Copyright (C) 2012 ACS. (e) Bright-field images of a carbon-layered electrode array (CLEAR) device implanted on the cerebral cortex of a mouse (top) and the device with transparent graphene electrode sites and traces on a Parylene C substrate (bottom). Scale bars, $500 \mu \mathrm{m}$ (f) Neural signal potentials recorded by the CLEAR device. $\mathrm{x}$-scale bars: $50 \mathrm{~ms}$, y-scale bars: $100 \mu \mathrm{V}$. Inset photograph shows a 473-nm light stimulus with an optical fiber through the CLEAR device implanted on the cortex of a mouse. (e,f) Reprinted with permission from [15], copyright 2012, NPG.

Figure 4. Field-effect transistors and optical resonators. (a) Schematic illustration of monolayer $\mathrm{MoS}_{2}$ based FET biosensor. The $\mathrm{MoS}_{2}$ channel is functionalized with probing biomolecules for detecting target biomolecules. Reprinted with permission from [51], Copyright (C) 2014 American Chemical Society (ACS). (b) Illustration (left) and image (right) of a graphene FET array with microfluidic channels on top.

The bottom inset shows a confocal fluorescence image of the array. Note stronger fluorescence intensity at even-numbered graphene sites with the site-specific probe DNA immobilization than that addnumbered sites without probe DNA. Reprinted with permission from [178], copyright @ 2014 , NPG. (c) Schematic illustration of a bioelectronic nose, based on a modified bilayer graphene (MBLG) conjugated with a human olfactory receptor. (d) Schematic diagram of a solution-gated FET B-nose using the MBLG. (e) Temporal responses (red/blue) and a calibration curve (black) of FET B-nose at varying concentrations (0.04 fM to $4 \mathrm{nM}$ ) of particular odorant amyl butyrate (AB). (c-e) Reprinted with permission from [88], Copyright (C) 2012 ACS. (f) Schematic illustration of a silicon nanowire (SiNW) array on a glass substrate. Monolayer graphene on the SiNW array facilitates the adsorption of target biomolecules. (g) Resonant wavelength shift in specific binding of IgG antibody on graphene for varying concentrations. (f, g) Reprinted with permission from [19], Copyright @ 2014 ACS. 
Figure 5. Nanopore-based sensing. (a) Schematic illustration of a graphene membrane mounted on an aperture $(200 \times 200 \mathrm{~nm})$ in $\mathrm{SiNx} / \mathrm{Si}$ frame. The membrane separates two ionic solutions in contact with $\mathrm{Ag} / \mathrm{AgCl}$ electrodes. The inset shows cross-section through the $\mathrm{Si}$ frame, $\mathrm{SiN}_{\mathrm{x}}$ aperture, and the drilled graphene membrane. The transmission electron microscope image on the right side shows the mounted graphene membrane with a nanopores ( $D \sim 8 \mathrm{~nm}$ ). Reprinted with permission from [7], copyright 2010, NPG. (b) Schematic illustration of a $\mathrm{MoS}_{2}$ nanopore membrane for DNA translocation, suspended on a $\mathrm{SiN}_{\mathrm{x}}$ membrane. DNA molecules are driven through a nanopore on $\mathrm{MoS}_{2}$ by an applied electrical field, and the ionic current through the pore is recorded. The inset TEM image shows a nanopore obtained by a focused electron beam. Reprinted with permission from [94], Copyright (C) 2014 ACS. (c) Illustration of a photothermal method of creating graphene nanopores: (I) Light is illuminated on a gold nanorod on graphene to fuse the nanorod and (II) the nanorod is reshaped into a hemispherical nanoparticle, and (III) the heated nanoparticle creates a nanopore on the graphene surface. (d) SEM images of nanopores created at different laser illumination power levels. (c, d) Reprinted with permission from [98], Copyright @ 2014 ACS.

Figure 6. Graphene multi-electrode arrays. (a) Schematic illustration of a graphene-based, carbonlayered electrode array (CLEAR) device with the layered structures. The inset optical image shows closeup of transparent graphene electrode sites on a parylene C substrate. (b) Demonstration of flexibility of the CLEAR device wrapped around a glass $\operatorname{rod}(R=2.9 \mathrm{~mm})$. Scale bar, $500 \mu \mathrm{m}$. (a, b) Reprinted with permission from [15], copyright (C) 2014, NPG. (c) Structure of the graphene MEAs. The inset shows an optical image of a graphene MEA on a $\mathrm{SiO}_{2} / \mathrm{Si}$ substrate. Scale bar, $100 \mu \mathrm{m}$. (d) Schematic illustration of a graphene microelectrode recording the action potential of an electrogenic cell. (c, d) Reprinted with permission from [106], Copyright (C) 2015, Springer Science+Business Media Dordrecht. 
Figure 7. Multi-functional sensor arrays. (a) Images of a 3D multifunctional integumentary membrane (3D-MIM) integrated on a rabbit heart. The inset image shows conformal integration on the heart surface. Scale bars, $6 \mathrm{~mm}$. (b) Magnified images of the functional elements noted with white arrows in (a). Scale bars, $500 \mu \mathrm{m}$. (a, b) Reprinted with permission from [73], copyright (c) 2014, NPG. (c) Schematic illustration of an epidermal electronics system (EES). (d) Image of EES laminated on wound and contralateral (control) sites. (e) Image of EES mounted on the forearm. (c-e) Reprinted from [75] with permission of John Wiley \& Sons, Inc.

Figure 8. Strain engineering of 2D materials and stretchable/biodegradable devices. (a) Schematic illustration of thermal shrinkage of polystyrene (PS) substrate to induce the crumpling of graphene (top), and atomic force microscope (AFM) image of uniaxially crumpled graphene (bottom). (b) SEM images of a FET device comprising a crumpled graphene channel and crumpled gold source/drain electrodes. (a, b) Reprinted with permission from [192], Copyright (C) 2015 ACS. (c) Schematic illustration of surfaceenhanced Raman scattering (SERS) enhancement from a crumpled graphene-gold nanoparticles (AuNPs) hybrid structure. Strong Raman spectral signal is detected when the target molecules are moved in the middle of AuNPs on crumpled graphene (inset). (d) Photograph (top) of the 3D crumpled graphene-Au NPs hybrid film (a dark square) on a PS substrate. SEM image (bottom) of the substrate. (c, d) Reprinted with permission from [194], Copyright (C) 2015 ACS. (e) Schematic illustration and photograph (inset) of stress-absorbing design using patterned, adhesive gel. (f) Photographs of a matrix coupled with adhesive gel electrodes (left), and the matrix device with stable contact on the surface of a balloon under $100 \%$ compressive strain. (right) Scale bars, $5 \mathrm{~mm}$. (e, f) Reprinted with permission from [80], copyright (C) 2014, NPG. (g) Photograph (top) and magnified photograph (bottom) of a heterojunction bipolar transistor (HBT) array on a cellulose nanofibril substrate on a tree leaf. (h) Photographs (top) and magnified photographs (bottom) showing degradation process at $6 \mathrm{~h}$ and 60 days. Notably, the array device is fully covered by fungus after 60 days. $(\mathrm{g}, \mathrm{h})$ Reprinted with permission from [72], copyright $($ ) 
Review Article in Microelectronic Engineering

2015, NPG.

\section{Figures}

Figure 1.
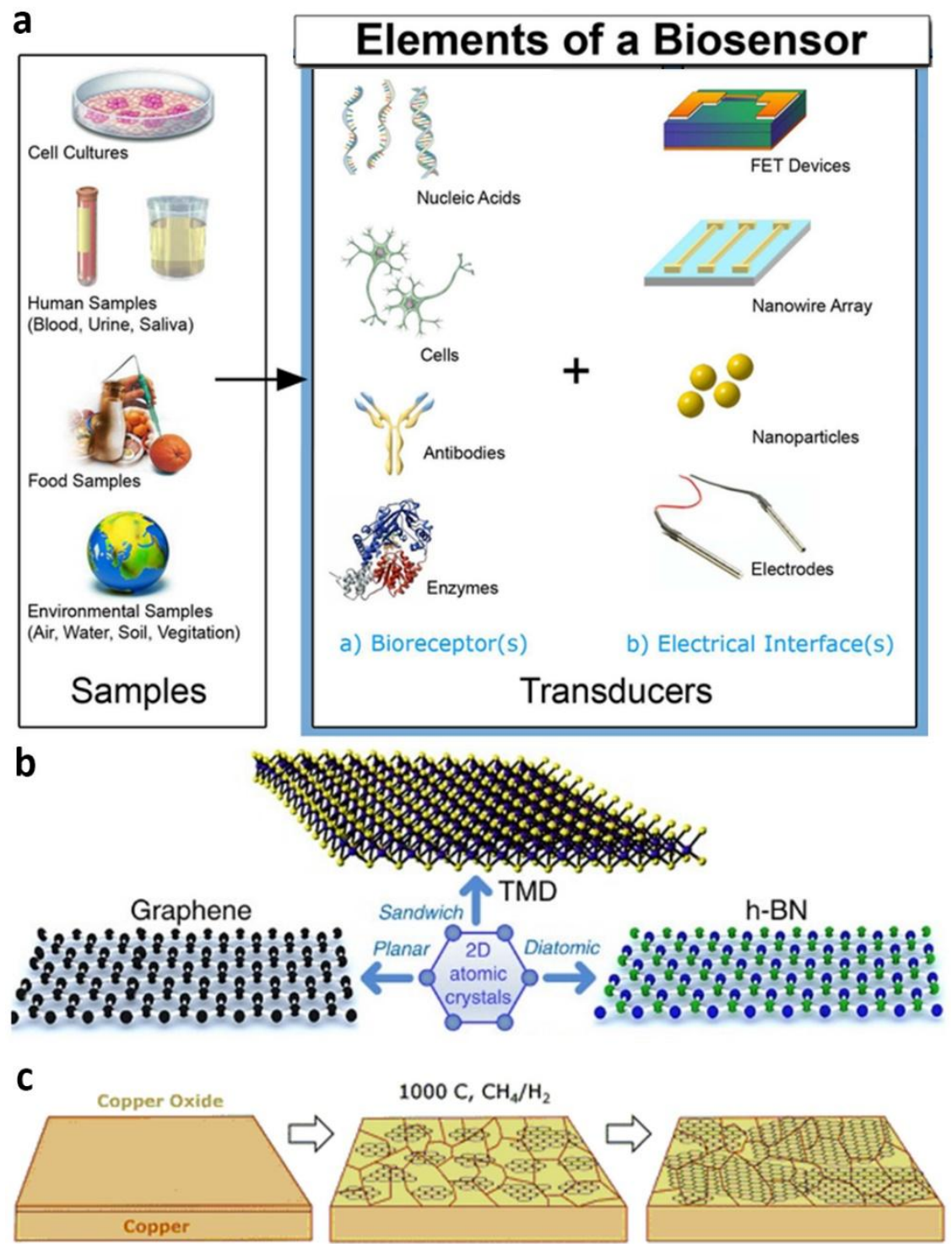
Review Article in Microelectronic Engineering

Figure 2.

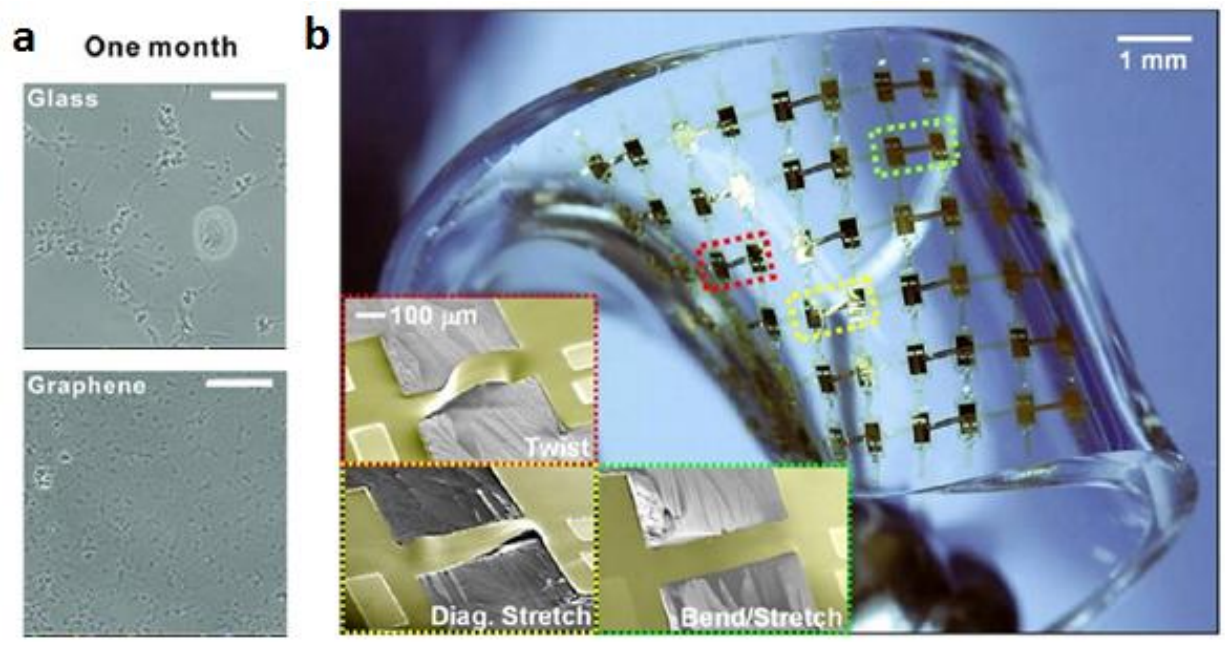


Figure 3.

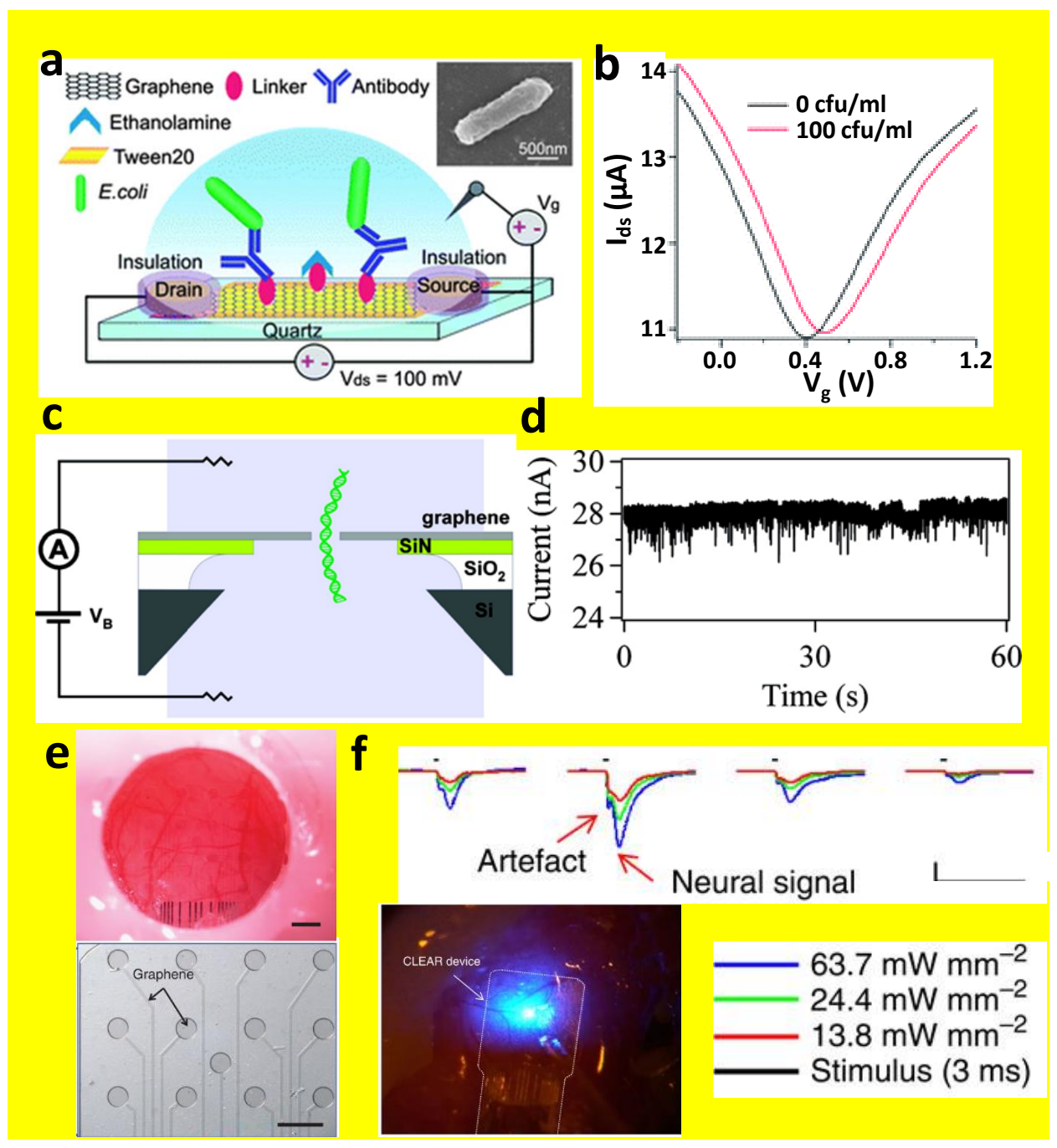


Review Article in Microelectronic Engineering

Figure 4.
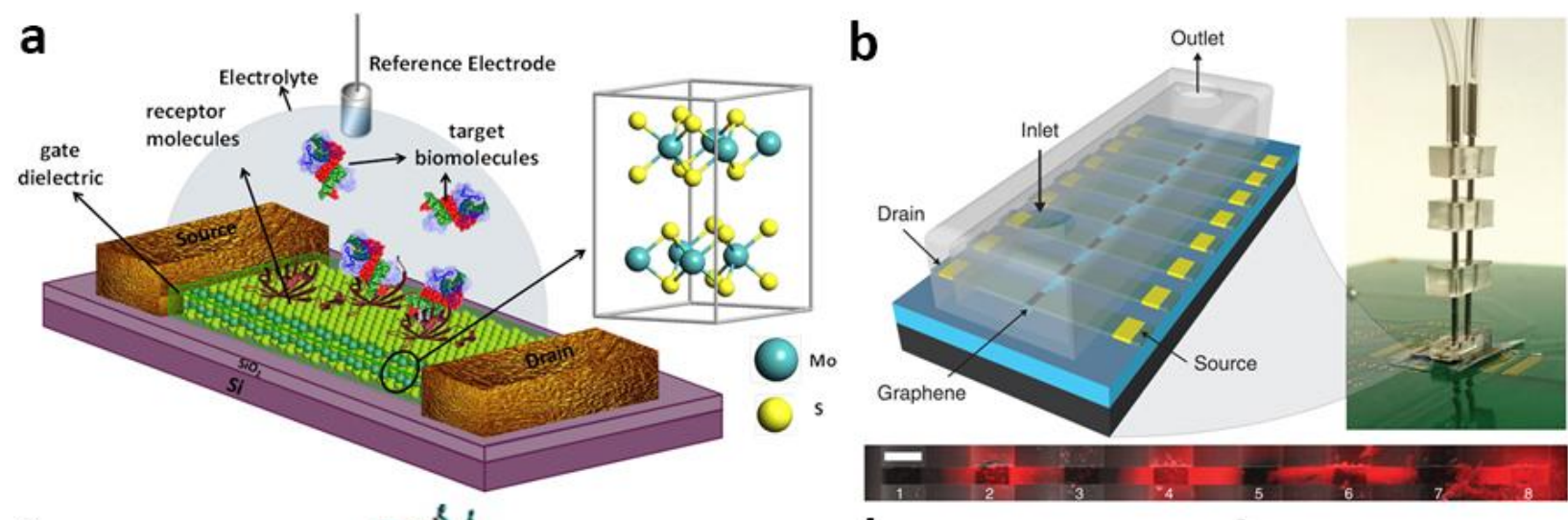

C
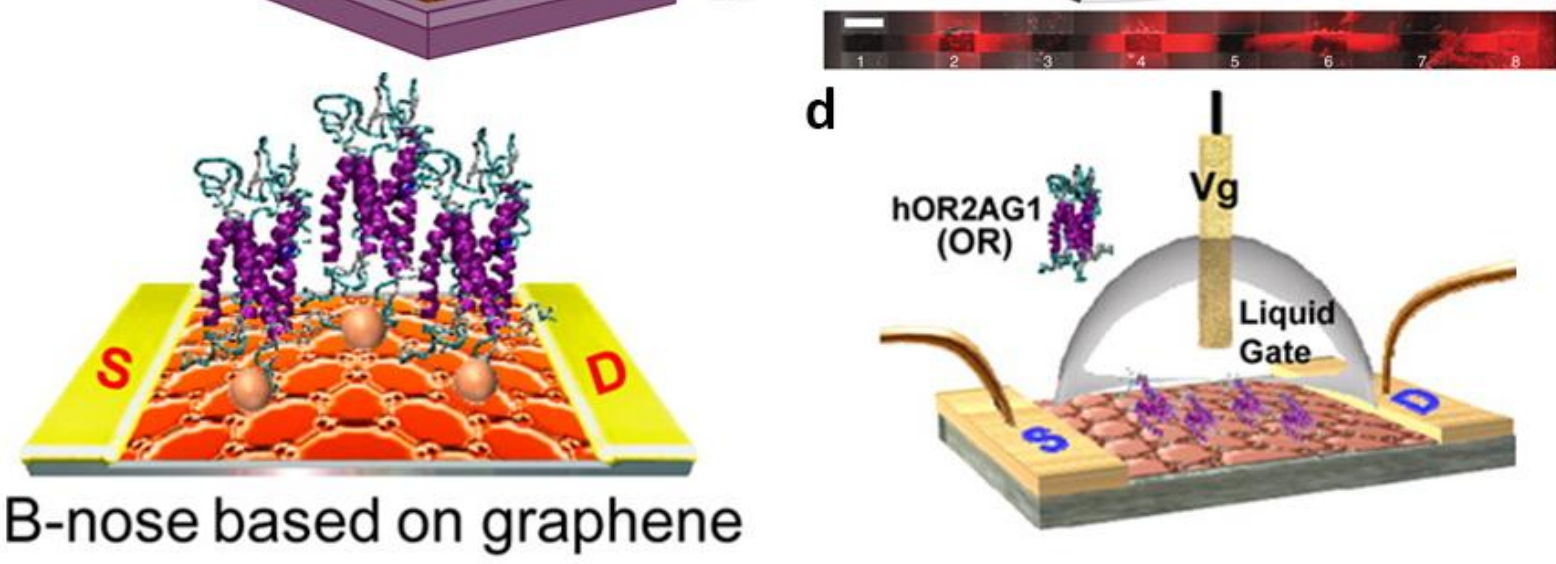

d
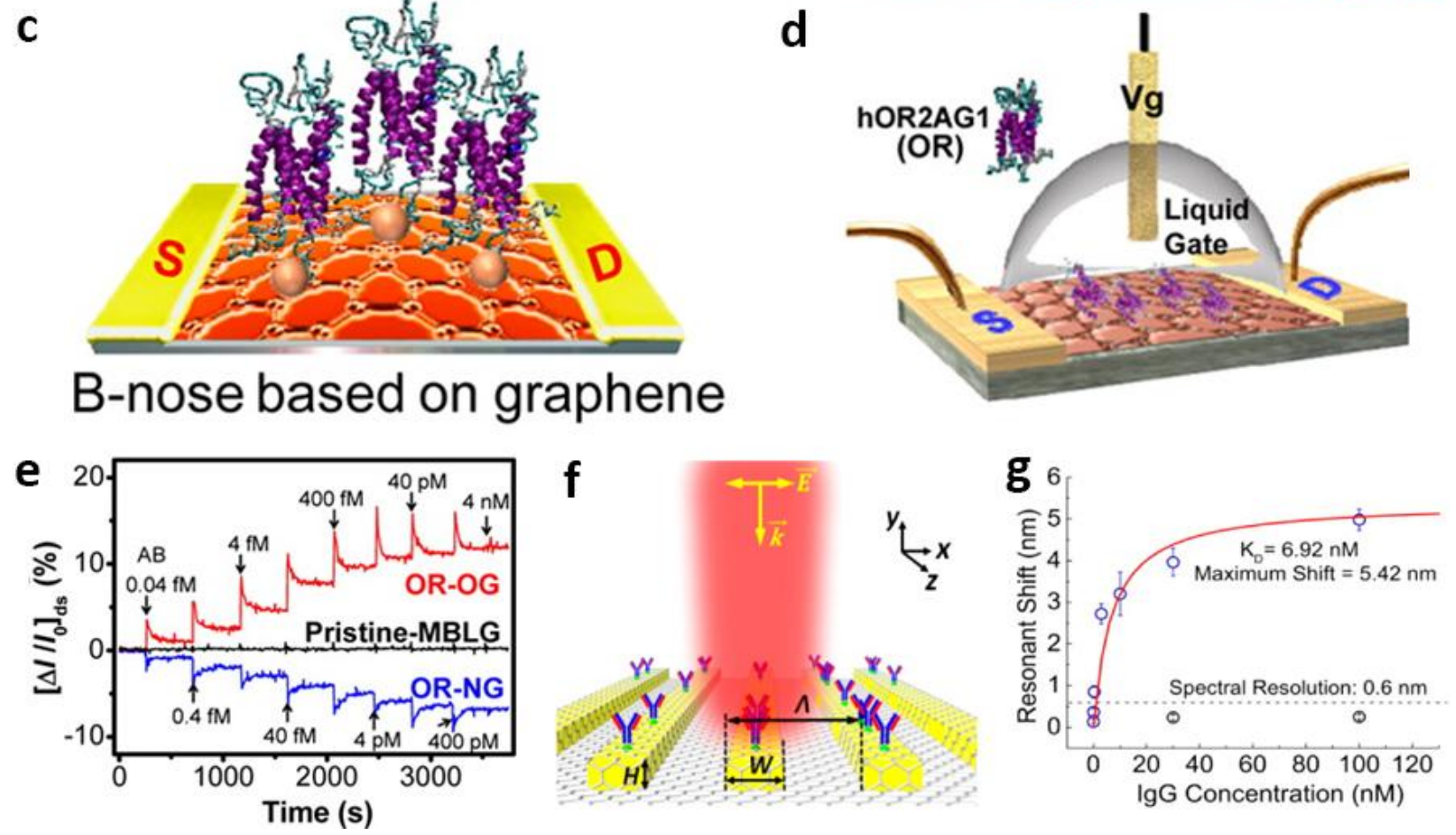
Figure 5.
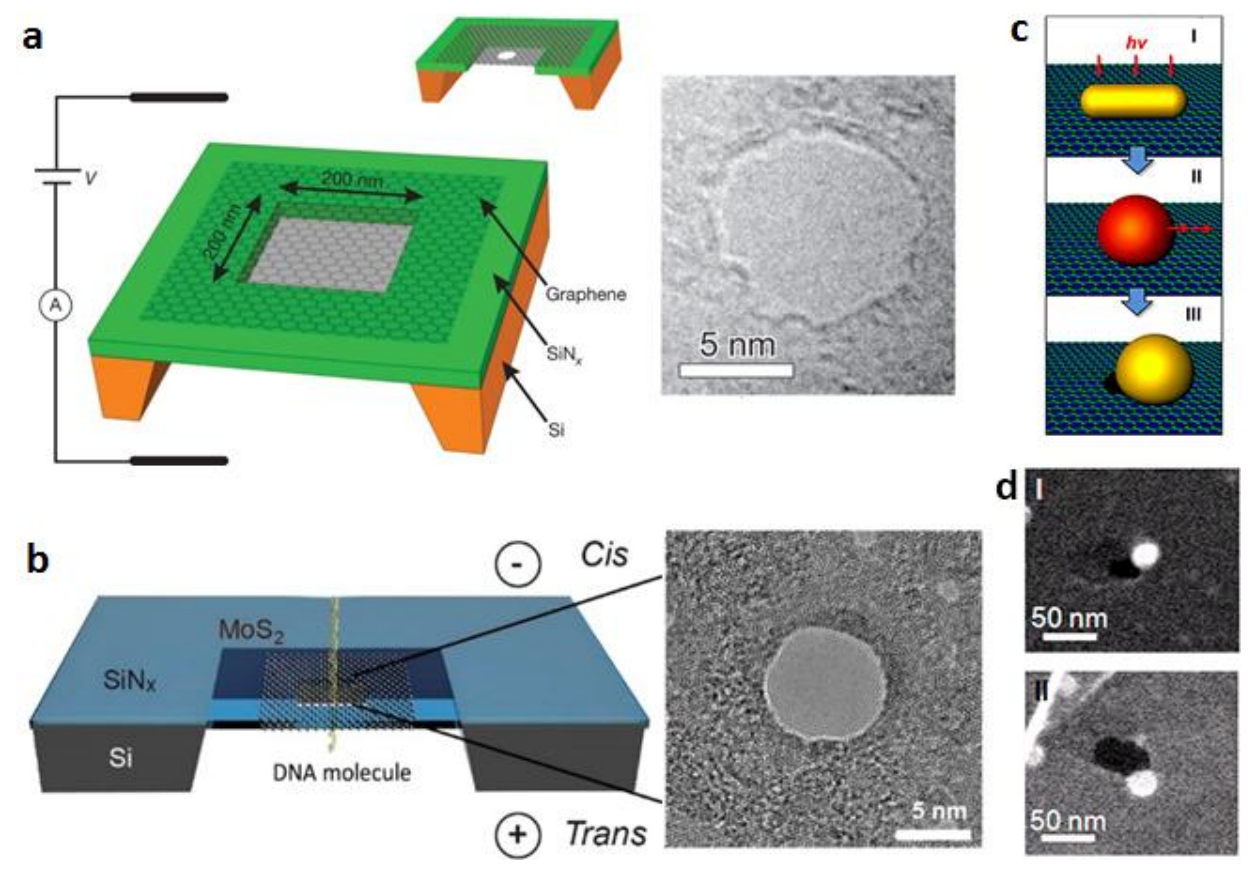
Figure 6.
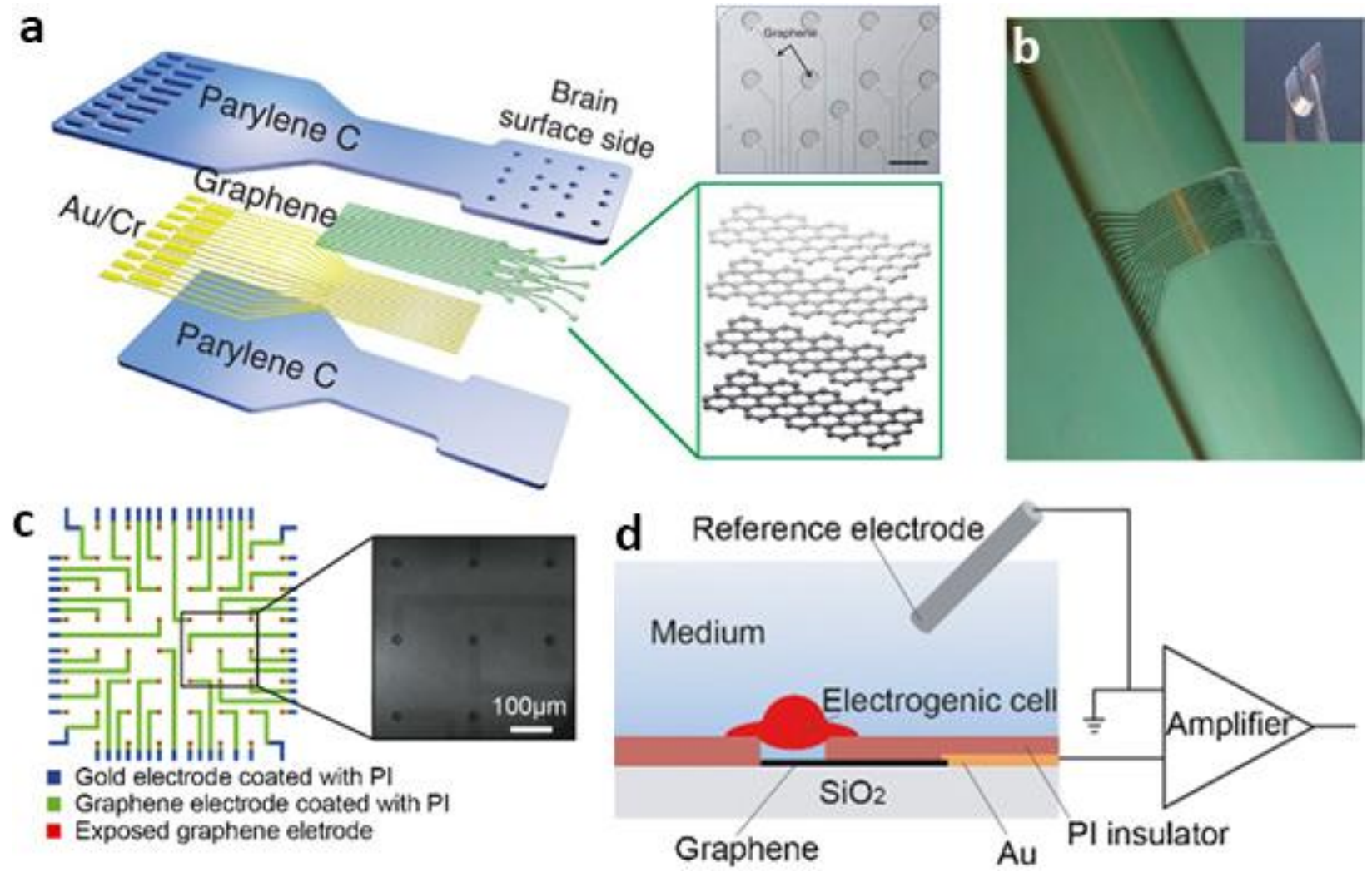
Figure 7.

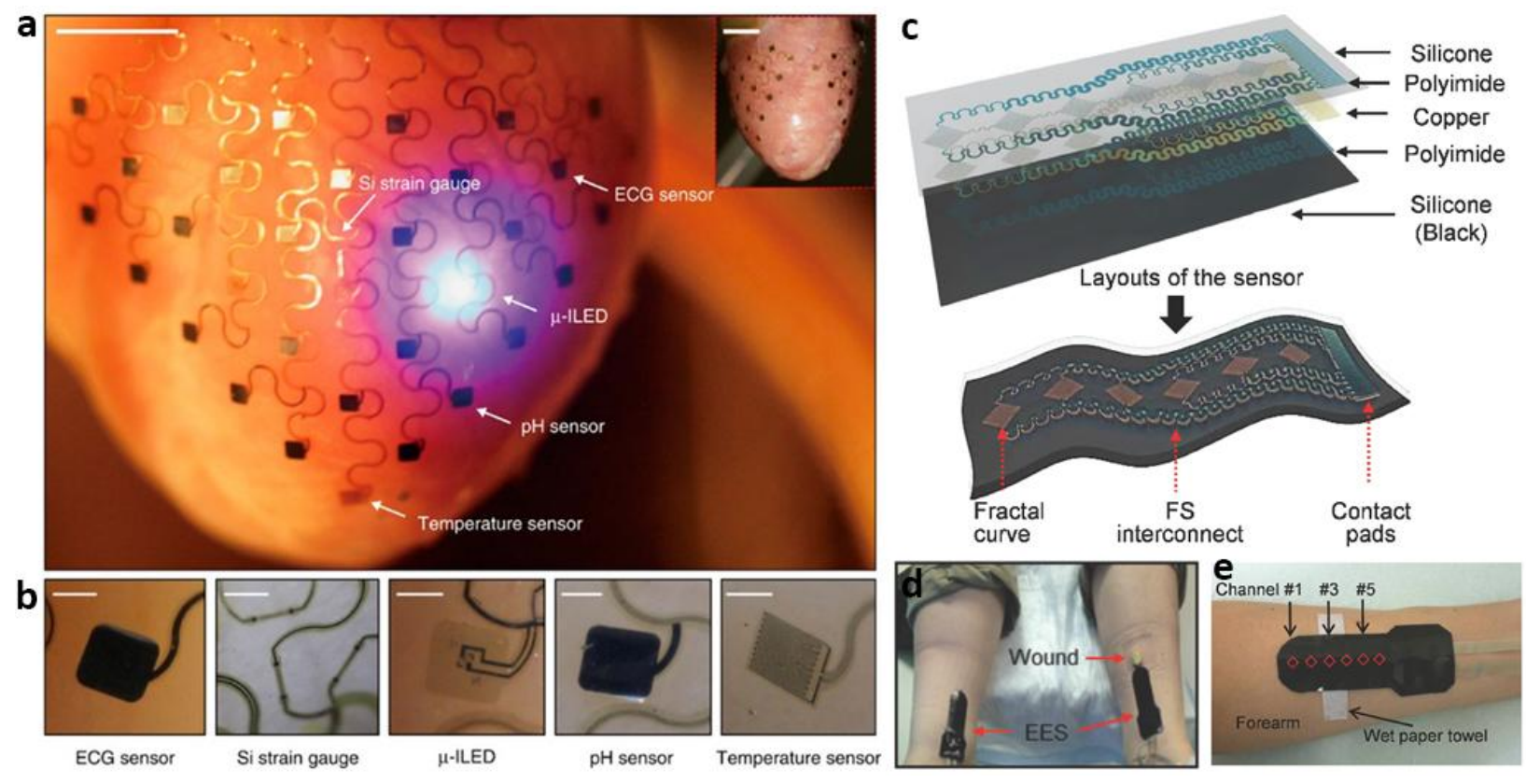


Figure 8.

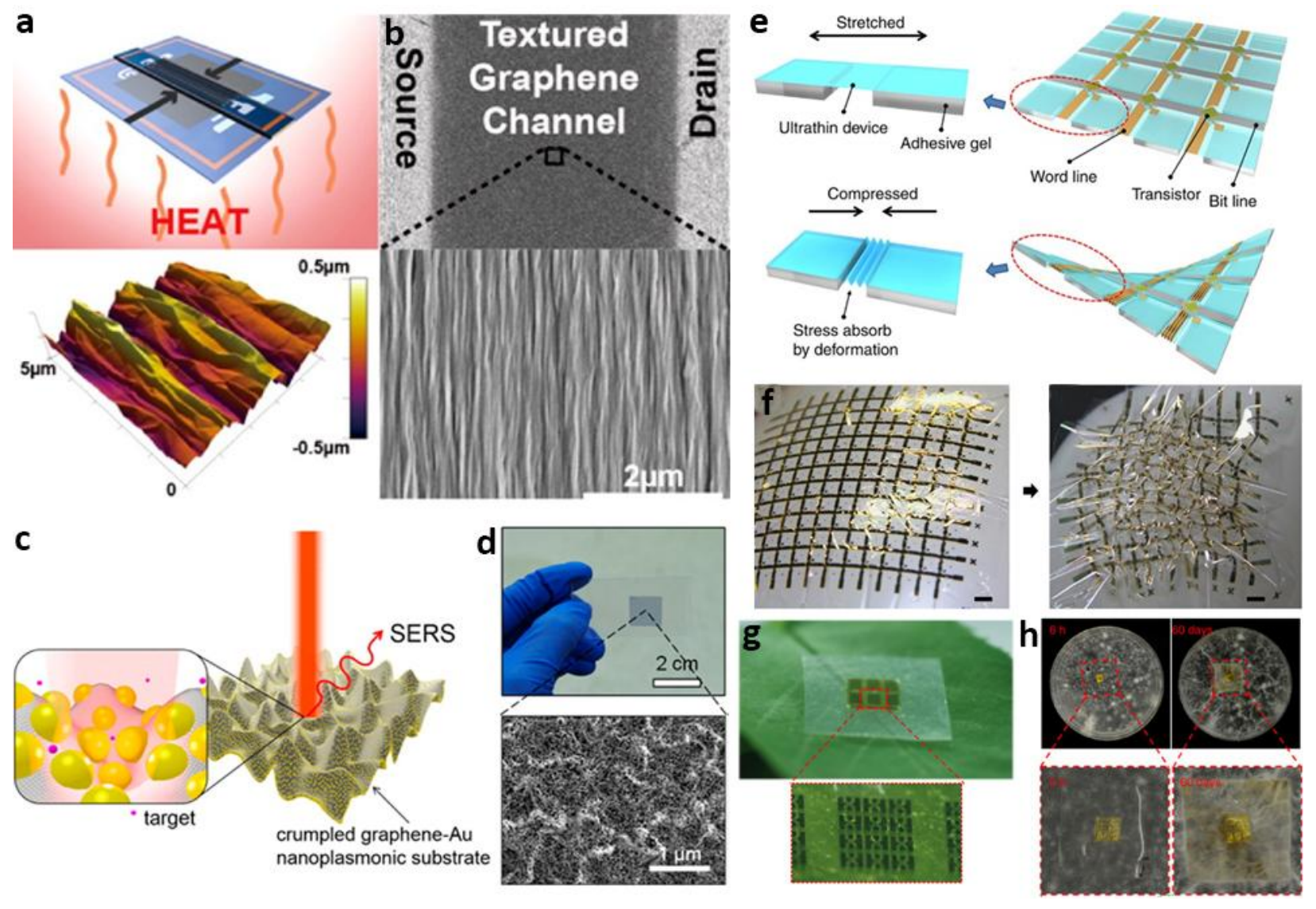




\section{References}

[1] G. Fiori, F. Bonaccorso, G. Iannaccone, T. Palacios, D. Neumaier, A. Seabaugh, S.K. Banerjee, L. Colombo, Electronics based on two-dimensional materials, Nat Nanotechnol, 9 (2014) 768-779.

[2] K.S. Novoselov, A.K. Geim, S.V. Morozov, D. Jiang, Y. Zhang, S.V. Dubonos, I.V. Grigorieva, A.A. Firsov, Electric Field Effect in Atomically Thin Carbon Films, Science, 306 (2004) 666-669.

[3] R. Stine, S.P. Mulvaney, J.T. Robinson, C.R. Tamanaha, P.E. Sheehan, Fabrication, Optimization, and Use of Graphene Field Effect Sensors, Anal Chem, 85 (2013) 509-521.

[4] L. Chen, J. Xie, H. Yoon, M. Srivatsan, R.E. Harbaugh, V.K. Varadan, Biohybrid Circuits: Nanotransducers Linking Cells and Neural Electrodes, Biohybrid Systems, Wiley-VCH Verlag GmbH \& Co. KGaA (2011) pp. 95-113.

[5] H.J. Du, J.S. Ye, J.Q. Zhang, X.D. Huang, C.Z. Yu, A voltammetric sensor based on graphenemodified electrode for simultaneous determination of catechol and hydroquinone, $\mathrm{J}$ Electroanal Chem, 650 (2011) 209-213.

[6] T.A. Gan, C.G. Hu, Z.L. Chen, S.S. Hu, Fabrication and application of a novel plant hormone sensor for the determination of methyl jasmonate based on self-assembling of phosphotungstic acid-graphene oxide nanohybrid on graphite electrode, Sensor Actuat B-Chem, 151 (2010) 8-14.

[7] S. Garaj, W. Hubbard, A. Reina, J. Kong, D. Branton, J.A. Golovchenko, Graphene as a subnanometre trans-electrode membrane, Nature, 467 (2010) 190-193.

[8] M.B. Gholivand, M. Khodadadian, Amperometric cholesterol biosensor based on the direct electrochemistry of cholesterol oxidase and catalase on a graphene/ionic liquid-modified glassy carbon electrode, Biosens Bioelectron, 53 (2014) 472-478.

[9] J. Ping, J. Wu, Y. Wang, Y. Ying, Simultaneous determination of ascorbic acid, dopamine and uric acid using high-performance screen-printed graphene electrode, Biosens Bioelectron, 34 (2012) 70-76.

[10] L. Bareket-Keren, Y. Hanein, Carbon nanotube-based multi electrode arrays for neuronal interfacing: progress and prospects, Front Neural Circuit, 6 (2012) 122.

[11] P. Connolly, P. Clark, A.S.G. Curtis, J.A.T. Dow, C.D.W. Wilkinson, An Extracellular Microelectrode Array for Monitoring Electrogenic Cells in Culture, Biosens Bioelectron, 5 (1990) 223-234.

[12] R. Huys, D. Braeken, D. Jans, A. Stassen, N. Collaert, J. Wouters, J. Loo, S. Severi, F. Vleugels, G. Callewaert, K. Verstreken, C. Bartic, W. Eberle, Single-cell recording and stimulation with a 16k micro-nail electrode array integrated on a 0.18 mu m CMOS chip, Lab Chip, 12 (2012) 12741280.

[13] F. Li, M. Xue, X. Ma, M. Zhang, T. Cao, Facile patterning of reduced graphene oxide film into microelectrode array for highly sensitive sensing, Anal Chem, 83 (2011) 6426-6430.

[14] A.M. Ng, Kenry, C. Teck Lim, H.Y. Low, K.P. Loh, Highly sensitive reduced graphene oxide microelectrode array sensor, Biosens Bioelectron, 65 (2014) 265-273.

[15] D.W. Park, A.A. Schendel, S. Mikael, S.K. Brodnick, T.J. Richner, J.P. Ness, M.R. Hayat, F. Atry, S.T. Frye, R. Pashaie, S. Thongpang, Z.Q. Ma, J.C. Williams, Graphene-based carbonlayered electrode array technology for neural imaging and optogenetic applications, Nat Commun, 5 (2014).

[16] M.E. Spira, A. Hai, Multi-electrode array technologies for neuroscience and cardiology, Nat Nanotechnol, 8 (2013) 83-94.

[17] C.A. Thomas, Jr., P.A. Springer, G.E. Loeb, Y. Berwald-Netter, L.M. Okun, A miniature microelectrode array to monitor the bioelectric activity of cultured cells, Exp Cell Res, 74 (1972) 61-66.

[18] B.C. Wheeler, Y. Nam, In Vitro Microelectrode Array Technology and Neural Recordings, Crit Rev Biomed Eng, 39 (2011) 45-61.

[19] Q. Guo, H. Zhu, F. Liu, A.Y. Zhu, J.C. Reed, F. Yi, E. Cubukcu, Silicon-on-Glass Graphene- 
Functionalized Leaky Cavity Mode Nanophotonic Biosensor, ACS Photonics, 1 (2014) 221-227.

[20] E. Petryayeva, U.J. Krull, Localized surface plasmon resonance: Nanostructures, bioassays and biosensing-A review, Anal Chim Acta, 706 (2011) 8-24.

[21] D. Rodrigo, O. Limaj, D. Janner, D. Etezadi, F.J. García de Abajo, V. Pruneri, H. Altug, Midinfrared plasmonic biosensing with graphene, Science, 349 (2015) 165-168.

[22] K.A. Willets, R.P. Van Duyne, Localized surface plasmon resonance spectroscopy and sensing, Annu Rev Phys Chem, 58 (2007) 267-297.

[23] L. Wu, H.S. Chu, W.S. Koh, E.P. Li, Highly sensitive graphene biosensors based on surface plasmon resonance, Opt Express, 18 (2010) 14395-14400.

[24] T. Kuila, S. Bose, P. Khanra, A.K. Mishra, N.H. Kim, J.H. Lee, Recent advances in graphenebased biosensors, Biosens Bioelectron, 26 (2011) 4637-4648.

[25] H.M. Ma, D. Wu, Z.T. Cui, Y. Li, Y. Zhang, B. Du, Q. Wei, Graphene-Based Optical and Electrochemical Biosensors: A Review, Anal Lett, 46 (2013) 1-17.

[26] Y.Y. Shao, J. Wang, H. Wu, J. Liu, I.A. Aksay, Y.H. Lin, Graphene Based Electrochemical Sensors and Biosensors: A Review, Electroanal, 22 (2010) 1027-1036.

[27] N. Choudhary, S. Hwang, W. Choi, B. Bhushan, D. Luo, S.R. Schricker, W. Sigmund, S. Zauscher, Carbon Nanomaterials: A Review, Springer Materials (2014) 709-769.

[28] A.K. Geim, I.V. Grigorieva, Van der Waals heterostructures, Nature, 499 (2013) 419-425.

[29] A.K. Geim, K.S. Novoselov, The rise of graphene, Nat Mater, 6 (2007) 183-191.

[30] S. Goenka, V. Sant, S. Sant, Graphene-based nanomaterials for drug delivery and tissue engineering, J Control Release, 173 (2014) 75-88.

[31] S.-W. Hwang, G. Park, C. Edwards, E.A. Corbin, S.-K. Kang, H. Cheng, J.-K. Song, J.-H. Kim, S. Yu, J. Ng, J.E. Lee, J. Kim, C. Yee, B. Bhaduri, Y. Su, F.G. Omennetto, Y. Huang, R. Bashir, L. Goddard, G. Popescu, K.-M. Lee, J.A. Rogers, Dissolution Chemistry and Biocompatibility of Single-Crystalline Silicon Nanomembranes and Associated Materials for Transient Electronics, ACS Nano, 8 (2014) 5843-5851.

[32] M. Irimia-Vladu, P.A. Troshin, M. Reisinger, L. Shmygleva, Y. Kanbur, G. Schwabegger, M. Bodea, R. Schwödiauer, A. Mumyatov, J.W. Fergus, V.F. Razumov, H. Sitter, N.S. Sariciftci, S. Bauer, Biocompatible and Biodegradable Materials for Organic Field-Effect Transistors, Adv Funct Mater, 20 (2010) 4069-4076.

[33] D. Jariwala, V.K. Sangwan, L.J. Lauhon, T.J. Marks, M.C. Hersam, Carbon nanomaterials for electronics, optoelectronics, photovoltaics, and sensing, Chem Soc Rev, 42 (2013) 2824-2860.

[34] S.H. Ku, M. Lee, C.B. Park, Carbon-Based Nanomaterials for Tissue Engineering, Adv Healthc Mater, 2 (2013) 244-260.

[35] S.K. Lee, H. Kim, B.S. Shim, Graphene: an emerging material for biological tissue engineering, Carbon Lett, 14 (2013) 63-75.

[36] S. Liu, X.F. Guo, Carbon nanomaterials field-effect-transistor-based biosensors, NPG Asia Mater, 4 (2012) 1-10.

[37] Y.X. Liu, X.C. Dong, P. Chen, Biological and chemical sensors based on graphene materials, Chem Soc Rev, 41 (2012) 2283-2307.

[38] F. Menaa, A. Abdelghani, B. Menaa, Graphene nanomaterials as biocompatible and conductive scaffolds for stem cells: impact for tissue engineering and regenerative medicine, $\mathrm{J}$ Tissue Eng Regen Med (2014).

[39] J. Ping, Y. Zhou, Y. Wu, V. Papper, S. Boujday, R.S. Marks, T.W. Steele, Recent advances in aptasensors based on graphene and graphene-like nanomaterials, Biosens Bioelectron, 64 (2015) 373-385.

[40] A.M. Pinto, I.C. Goncalves, F.D. Magalhaes, Graphene-based materials biocompatibility: A review, Colloids Surf B, 111 (2013) 188-202.

[41] J.A. Rogers, T. Someya, Y.G. Huang, Materials and Mechanics for Stretchable Electronics, Science, 327 (2010) 1603-1607. 
[42] Y. Song, Y. Luo, C. Zhu, H. Li, D. Du, Y. Lin, Recent advances in electrochemical biosensors based on graphene two-dimensional nanomaterials, Biosens Bioelectron, 76 (2015) 195-212.

[43] I. Willner, Biomaterials for sensors, fuel cells, and circuitry, Science, 298 (2002) 2407-2408.

[44] W. Yuan, J. Chen, G. Shi, Nanoporous graphene materials, Mater Today, 17 (2014) 77-85.

[45] Y. Zhao, Y. Xie, Z. Liu, X. Wang, Y. Chai, F. Yan, Two-dimensional material membranes: an emerging platform for controllable mass transport applications, Small, 10 (2014) 4521-4542.

[46] S. Bae, H. Kim, Y. Lee, X. Xu, J.S. Park, Y. Zheng, J. Balakrishnan, T. Lei, H.R. Kim, Y.I. Song, Y.J. Kim, K.S. Kim, B. Ozyilmaz, J.H. Ahn, B.H. Hong, S. Iijima, Roll-to-roll production of 30inch graphene films for transparent electrodes, Nat Nanotechnol, 5 (2010) 574-578.

[47] R.R. Nair, P. Blake, A.N. Grigorenko, K.S. Novoselov, T.J. Booth, T. Stauber, N.M.R. Peres, A.K. Geim, Fine Structure Constant Defines Visual Transparency of Graphene, Science, 320 (2008) 1308.

[48] C. Lee, X. Wei, J.W. Kysar, J. Hone, Measurement of the Elastic Properties and Intrinsic Strength of Monolayer Graphene, Science, 321 (2008) 385-388.

[49] S.K. Lee, H.Y. Jang, S. Jang, E. Choi, B.H. Hong, J. Lee, S. Park, J.H. Ahn, All graphene-based thin film transistors on flexible plastic substrates, Nano Lett, 12 (2012) 3472-3476.

[50] H. Wu, R. Yang, B. Song, Q. Han, J. Li, Y. Zhang, Y. Fang, R. Tenne, C. Wang, Biocompatible Inorganic Fullerene-Like Molybdenum Disulfide Nanoparticles Produced by Pulsed Laser Ablation in Water, ACS Nano, 5 (2011) 1276-1281.

[51] D. Sarkar, W. Liu, X.J. Xie, A.C. Anselmo, S. Mitragotri, K. Banerjee, $\mathrm{MoS}_{2}$ Field-Effect Transistor for Next-Generation Label-Free Biosensors, ACS Nano, 8 (2014) 3992-4003.

[52] C.J. Bettinger, Z.A. Bao, Organic Thin-Film Transistors Fabricated on Resorbable Biomaterial Substrates, Adv Mater, 22 (2010) 651-655.

[53] D. Akinwande, N. Petrone, J. Hone, Two-dimensional flexible nanoelectronics, Nat Commun, 5 (2014) 5678.

[54] A. Nathan, A. Ahnood, M.T. Cole, S. Lee, Y. Suzuki, P. Hiralal, F. Bonaccorso, T. Hasan, L. Garcia-Gancedo, A. Dyadyusha, S. Haque, P. Andrew, S. Hofmann, J. Moultrie, D.P. Chu, A.J. Flewitt, A.C. Ferrari, M.J. Kelly, J. Robertson, G.A.J. Amaratunga, W.I. Milne, Flexible Electronics: The Next Ubiquitous Platform, Proc IEEE, 100 (2012) 1486-1517.

[55] D.-H. Kim, J. Viventi, J.J. Amsden, J. Xiao, L. Vigeland, Y.-S. Kim, J.A. Blanco, B. Panilaitis, E.S. Frechette, D. Contreras, D.L. Kaplan, F.G. Omenetto, Y. Huang, K.-C. Hwang, M.R. Zakin, B. Litt, J.A. Rogers, Dissolvable films of silk fibroin for ultrathin conformal bio-integrated electronics, Nat Mater, 9 (2010) 511-517.

[56] Y.J. Park, S.K. Lee, M.S. Kim, H. Kim, J.H. Ahn, Graphene-Based Conformal Devices, ACS Nano, 8 (2014) 7655-7662.

[57] K.I. Chen, B.R. Li, Y.T. Chen, Silicon nanowire field-effect transistor-based biosensors for biomedical diagnosis and cellular recording investigation, Nano Today, 6 (2011) 131-154.

[58] D. Grieshaber, R. MacKenzie, J. Vörös, E. Reimhult, Electrochemical Biosensors - Sensor Principles and Architectures, Sensors, 8 (2008) 1400.

[59] S.W. Hwang, J.K. Song, X. Huang, H.Y. Cheng, S.K. Kang, B.H. Kim, J.H. Kim, S. Yu, Y.G. Huang, J.A. Rogers, High-Performance Biodegradable/Transient Electronics on Biodegradable Polymers, Adv Mater, 26 (2014) 3905-3911.

[60] S.W. Hwang, H. Tao, D.H. Kim, H.Y. Cheng, J.K. Song, E. Rill, M.A. Brenckle, B. Panilaitis, S.M. Won, Y.S. Kim, Y.M. Song, K.J. Yu, A. Ameen, R. Li, Y.W. Su, M.M. Yang, D.L. Kaplan, M.R. Zakin, M.J. Slepian, Y.G. Huang, F.G. Omenetto, J.A. Rogers, A Physically Transient Form of Silicon Electronics, Science, 337 (2012) 1640-1644.

[61] F. Bonaccorso, A. Lombardo, T. Hasan, Z.P. Sun, L. Colombo, A.C. Ferrari, Production and processing of graphene and 2d crystals, Mater Today, 15 (2012) 564-589.

[62] L. Colombo, R.M. Wallace, R.S. Ruoff, Graphene Growth and Device Integration, Proc IEEE, 101 (2013) 1536-1556. 
[63] F. Schedin, A.K. Geim, S.V. Morozov, E.W. Hill, P. Blake, M.I. Katsnelson, K.S. Novoselov, Detection of individual gas molecules adsorbed on graphene, Nat Mater, 6 (2007) 652-655.

[64] K.S. Kim, Y. Zhao, H. Jang, S.Y. Lee, J.M. Kim, K.S. Kim, J.H. Ahn, P. Kim, J.Y. Choi, B.H. Hong, Large-scale pattern growth of graphene films for stretchable transparent electrodes, Nature, 457 (2009) 706-710.

[65] A.C. Ferrari, D.M. Basko, Raman spectroscopy as a versatile tool for studying the properties of graphene, Nat Nanotechnol, 8 (2013) 235-246.

[66] H.X. Chang, H.K. Wu, Graphene-based nanocomposites: preparation, functionalization, and energy and environmental applications, Energ Environ Sci, 6 (2013) 3483-3507.

[67] C. Mattevi, H. Kim, M. Chhowalla, A review of chemical vapour deposition of graphene on copper, J Mater Chem, 21 (2011) 3324-3334.

[68] A. Castellanos-Gomez, V. Singh, H.S.J. van der Zant, G.A. Steele, Mechanics of freelysuspended ultrathin layered materials, Annalen der Physik, 527 (2015) 27-44.

[69] A.C. Ferrari, F. Bonaccorso, V. Fal'ko, K.S. Novoselov, S. Roche, P. Boggild, S. Borini, F.H.L. Koppens, V. Palermo, N. Pugno, J.A. Garrido, R. Sordan, A. Bianco, L. Ballerini, M. Prato, E. Lidorikis, J. Kivioja, C. Marinelli, T. Ryhanen, A. Morpurgo, J.N. Coleman, V. Nicolosi, L. Colombo, A. Fert, M. Garcia-Hernandez, A. Bachtold, G.F. Schneider, F. Guinea, C. Dekker, M. Barbone, Z. Sun, C. Galiotis, A.N. Grigorenko, G. Konstantatos, A. Kis, M. Katsnelson, L. Vandersypen, A. Loiseau, V. Morandi, D. Neumaier, E. Treossi, V. Pellegrini, M. Polini, A. Tredicucci, G.M. Williams, B. Hee Hong, J.-H. Ahn, J. Min Kim, H. Zirath, B.J. van Wees, H. van der Zant, L. Occhipinti, A. Di Matteo, I.A. Kinloch, T. Seyller, E. Quesnel, X. Feng, K. Teo, N. Rupesinghe, P. Hakonen, S.R.T. Neil, Q. Tannock, T. Lofwander, J. Kinaret, Science and technology roadmap for graphene, related two-dimensional crystals, and hybrid systems, Nanoscale, 7 (2015) 4598-4810.

[70] X. Qian, Y. Wang, W. Li, J. Lu, J. Li, Modelling of stacked 2D materials and devices, 2D Materials, 2 (2015) 032003.

[71] D.-H. Kim, R. Ghaffari, N. Lu, S. Wang, S.P. Lee, H. Keum, R. D'Angelo, L. Klinker, Y. Su, C. Lu, Y.-S. Kim, A. Ameen, Y. Li, Y. Zhang, B. de Graff, Y.-Y. Hsu, Z. Liu, J. Ruskin, L. Xu, C. Lu, F.G. Omenetto, Y. Huang, M. Mansour, M.J. Slepian, J.A. Rogers, Electronic sensor and actuator webs for large-area complex geometry cardiac mapping and therapy, Proc Natl Acad Sci USA, 109 (2012) 19910-19915.

[72] Y.H. Jung, T.H. Chang, H.L. Zhang, C.H. Yao, Q.F. Zheng, V.W. Yang, H.Y. Mi, M. Kim, S.J. Cho, D.W. Park, H. Jiang, J. Lee, Y.J. Qiu, W.D. Zhou, Z.Y. Cai, S.Q. Gong, Z.Q. Ma, Highperformance green flexible electronics based on biodegradable cellulose nanofibril paper, Nat Commun, 6 (2015).

[73] L. Xu, S.R. Gutbrod, A.P. Bonifas, Y. Su, M.S. Sulkin, N. Lu, H.-J. Chung, K.-I. Jang, Z. Liu, M. Ying, C. Lu, R.C. Webb, J.-S. Kim, J.I. Laughner, H. Cheng, Y. Liu, A. Ameen, J.-W. Jeong, G.T. Kim, Y. Huang, I.R. Efimov, J.A. Rogers, 3D multifunctional integumentary membranes for spatiotemporal cardiac measurements and stimulation across the entire epicardium, Nat Commun, 5 (2014).

[74] C. Legnani, C. Vilani, V.L. Calil, H.S. Barud, W.G. Quirino, C.A. Achete, S.J.L. Ribeiro, M. Cremona, Bacterial cellulose membrane as flexible substrate for organic light emitting devices, Thin Solid Films, 517 (2008) 1016-1020.

[75] Y. Hattori, L. Falgout, W. Lee, S.Y. Jung, E. Poon, J.W. Lee, I. Na, A. Geisler, D. Sadhwani, Y. Zhang, Y. Su, X. Wang, Z. Liu, J. Xia, H. Cheng, R.C. Webb, A.P. Bonifas, P. Won, J.W. Jeong, K.I. Jang, Y.M. Song, B. Nardone, M. Nodzenski, J.A. Fan, Y. Huang, D.P. West, A.S. Paller, M. Alam, W.H. Yeo, J.A. Rogers, Multifunctional skin-like electronics for quantitative, clinical monitoring of cutaneous wound healing, Adv Healthc Mater, 3 (2014) 1597-1607.

[76] H. Jiang, Chemical preparation of graphene-based nanomaterials and their applications in chemical and biological sensors, Small, 7 (2011) 2413-2427. 
[77] S.Y. Park, J. Park, S.H. Sim, M.G. Sung, K.S. Kim, B.H. Hong, S. Hong, Enhanced Differentiation of Human Neural Stem Cells into Neurons on Graphene, Adv Mater, 23 (2011) H263-+.

[78] D.H. Kim, R. Ghaffari, N.S. Lu, J.A. Rogers, Flexible and Stretchable Electronics for Biointegrated Devices, Annu Rev Biomed Eng, 14 (2012) 113-128.

[79] D.H. Kim, J. Song, W.M. Choi, H.S. Kim, R.H. Kim, Z. Liu, Y.Y. Huang, K.C. Hwang, Y.W. Zhang, J.A. Rogers, Materials and noncoplanar mesh designs for integrated circuits with linear elastic responses to extreme mechanical deformations, Proc Natl Acad Sci USA, 105 (2008) 18675-18680.

[80] S. Lee, Y. Inoue, D. Kim, A. Reuveny, K. Kuribara, T. Yokota, J. Reeder, M. Sekino, T. Sekitani, Y. Abe, T. Someya, A strain-absorbing design for tissue-machine interfaces using a tunable adhesive gel, Nat Commun, 5 (2014).

[81] T. Cohen-Karni, Q. Qing, Q. Li, Y. Fang, C.M. Lieber, Graphene and Nanowire Transistors for Cellular Interfaces and Electrical Recording, Nano Lett, 10 (2010) 1098-1102.

[82] M. Dankerl, M.V. Hauf, A. Lippert, L.H. Hess, S. Birner, I.D. Sharp, A. Mahmood, P. Mallet, J.Y. Veuillen, M. Stutzmann, J.A. Garrido, Graphene Solution-Gated Field-Effect Transistor Array for Sensing Applications, Adv Funct Mater, 20 (2010) 3117-3124.

[83] X.C. Dong, Y.M. Shi, W. Huang, P. Chen, L.J. Li, Electrical Detection of DNA Hybridization with Single-Base Specificity Using Transistors Based on CVD-Grown Graphene Sheets, Adv Mater, 22 (2010) 1649-1653.

[84] L.H. Hess, M. Seifert, J.A. Garrido, Graphene Transistors for Bioelectronics, Proc IEEE, 101 (2013) 1780-1792.

[85] S. Mao, K.H. Yu, J.B. Chang, D.A. Steeber, L.E. Ocola, J.H. Chen, Direct Growth of Verticallyoriented Graphene for Field-Effect Transistor Biosensor, Sci Rep, 3 (2013).

[86] N. Mohanty, V. Berry, Graphene-Based Single-Bacterium Resolution Biodevice and DNA Transistor: Interfacing Graphene Derivatives with Nanoscale and Microscale Biocomponents, Nano Lett, 8 (2008) 4469-4476.

[87] Y. Ohno, K. Maehashi, K. Matsumoto, Label-Free Biosensors Based on Aptamer-Modified Graphene Field-Effect Transistors, J Am Chem Soc, 132 (2010) 18012-18013.

[88] S.J. Park, O.S. Kwon, S.H. Lee, H.S. Song, T.H. Park, J. Jang, Ultrasensitive Flexible Graphene Based Field-Effect Transistor (FET)-Type Bioelectronic Nose, Nano Lett, 12 (2012) 5082-5090.

[89] B. Radisavljevic, A. Radenovic, J. Brivio, V. Giacometti, A. Kis, Single-layer $\mathrm{MoS}_{2}$ transistors, Nat Nanotechnol, 6 (2011) 147-150.

[90] B.B. Zhan, C. Li, J. Yang, G. Jenkins, W. Huang, X.C. Dong, Graphene Field-Effect Transistor and Its Application for Electronic Sensing, Small, 10 (2014) 4042-4065.

[91] J. Feng, K. Liu, M. Graf, M. Lihter, R.D. Bulushev, D. Dumcenco, D.T. Alexander, D. Krasnozhon, T. Vuletic, A. Kis, A. Radenovic, Electrochemical Reaction in Single Layer $\mathrm{MoS}_{2}$ : Nanopores Opened Atom by Atom, Nano Lett, 15 (2015) 3431-3438.

[92] Y. He, M. Tsutsui, S. Ryuzaki, K. Yokota, M. Taniguchi, T. Kawai, Graphene/hexagonal boron nitride/graphene nanopore for electrical detection of single molecules, NPG Asia Mater, 6 (2014) e104.

[93] J. Larkin, R. Henley, D.C. Bell, T. Cohen-Karni, J.K. Rosenstein, M. Wanunu, Slow DNA Transport through Nanopores in Hafnium Oxide Membranes, Acs Nano, 7 (2013) 10121-10128.

[94] K. Liu, J. Feng, A. Kis, A. Radenovic, Atomically Thin Molybdenum Disulfide Nanopores with High Sensitivity for DNA Translocation, ACS Nano, 8 (2014) 2504-2511.

[95] S. Liu, B. Lu, Q. Zhao, J. Li, T. Gao, Y. Chen, Y. Zhang, Z. Liu, Z. Fan, F. Yang, L. You, D. Yu, Boron nitride nanopores: highly sensitive DNA single-molecule detectors, Adv Mater, 25 (2013) 4549-4554.

[96] B. McNally, A. Singer, Z.L. Yu, Y.J. Sun, Z.P. Weng, A. Meller, Optical Recognition of Converted DNA Nucleotides for Single-Molecule DNA Sequencing Using Nanopore Arrays, 
Nano Lett, 10 (2010) 2237-2244.

[97] C.A. Merchant, K. Healy, M. Wanunu, V. Ray, N. Peterman, J. Bartel, M.D. Fischbein, K. Venta, Z.T. Luo, A.T.C. Johnson, M. Drndic, DNA Translocation through Graphene Nanopores, Nano Lett, 10 (2010) 2915-2921.

[98] S. Nam, I. Choi, C.C. Fu, K. Kim, S. Hong, Y. Choi, A. Zettl, L.P. Lee, Graphene nanopore with a self-integrated optical antenna, Nano Lett, 14 (2014) 5584-5589.

[99] S.W. Nam, M.J. Rooks, K.B. Kim, S.M. Rossnagel, Ionic Field Effect Transistors with Sub-10 nm Multiple Nanopores, Nano Lett, 9 (2009) 2044-2048.

[100] F. Sawafta, B. Clancy, A.T. Carlsen, M. Huber, A.R. Hall, Solid-state nanopores and nanopore arrays optimized for optical detection, Nanoscale, 6 (2014) 6991-6996.

[101] J. Shi, J. Hou, Y. Fang, Recent advances in nanopore-based nucleic acid analysis and sequencing, Microchimica Acta (2015).

[102] X. Shi, R. Gao, Y.L. Ying, W. Si, Y. Chen, Y.T. Long, An integrated system for optical and electrical detection of single molecules/particles inside a solid-state nanopore, Farad Discuss, 184 (2015) 85-99.

[103] M. Wanunu, Nanopores: A journey towards DNA sequencing, Phys Life Rev, 9 (2012) 125-158.

[104] G.W. Gross, A.N. Williams, J.H. Lucas, Recording of spontaneous activity with photoetched microelectrode surfaces from mouse spinal neurons in culture, J Neurosci Methods, 5 (1982) 1322.

[105] D. Kuzum, H. Takano, E. Shim, J.C. Reed, H. Juul, A.G. Richardson, J. de Vries, H. Bink, M.A. Dichter, T.H. Lucas, D.A. Coulter, E. Cubukcu, B. Litt, Transparent and flexible low noise graphene electrodes for simultaneous electrophysiology and neuroimaging, Nat Commun, 5 (2014).

[106] X. Du, L. Wu, J. Cheng, S. Huang, Q. Cai, Q. Jin, J. Zhao, Graphene microelectrode arrays for neural activity detection, J Biol Phys, 41 (2015) 339-347.

[107] J. Viventi, D.H. Kim, L. Vigeland, E.S. Frechette, J.A. Blanco, Y.S. Kim, A.E. Avrin, V.R. Tiruvadi, S.W. Hwang, A.C. Vanleer, D.F. Wulsin, K. Davis, C.E. Gelber, L. Palmer, J. Van der Spiegel, J. Wu, J.L. Xiao, Y.G. Huang, D. Contreras, J.A. Rogers, B. Litt, Flexible, foldable, actively multiplexed, high-density electrode array for mapping brain activity in vivo, Nat Neurosci, 14 (2011) 1599-U1138.

[108] H. Liu, Y. Liu, D. Zhu, Chemical doping of graphene, J. Mater. Chem., 21 (2011) 3335-3345.

[109] M. Pumera, Graphene in biosensing, Mater Today, 14 (2011) 308-315.

[110] O. Akhavan, E. Ghaderi, Photocatalytic Reduction of Graphene Oxide Nanosheets on TiO2 Thin Film for Photoinactivation of Bacteria in Solar Light Irradiation, J Phys Chem C, 113 (2009) 20214-20220.

[111] B. Fan, W. Li, Miniaturized optogenetic neural implants: a review, Lab Chip, 15 (2015) 38383855 .

[112] A.B. Artyukhin, M. Stadermann, R.W. Friddle, P. Stroeve, O. Bakajin, A. Noy, Controlled Electrostatic Gating of Carbon Nanotube FET Devices, Nano Lett, 6 (2006) 2080-2085.

[113] S. Boussaad, N.J. Tao, R. Zhang, T. Hopson, L.A. Nagahara, In situ detection of cytochrome c adsorption with single walled carbon nanotube device, Chem Commun, 13 (2003) 1502-1503.

[114] E.L. Gui, L.-J. Li, K. Zhang, Y. Xu, X. Dong, X. Ho, P.S. Lee, J. Kasim, Z.X. Shen, J.A. Rogers, Mhaisalkar, DNA Sensing by Field-Effect Transistors Based on Networks of Carbon Nanotubes, J Am Chem Soc, 129 (2007) 14427-14432.

[115] H.R. Byon, H.C. Choi, Network Single-Walled Carbon Nanotube-Field Effect Transistors (SWNT-FETs) with Increased Schottky Contact Area for Highly Sensitive Biosensor Applications, J Am Chem Soc, 128 (2006) 2188-2189.

[116] R.J. Chen, H.C. Choi, S. Bangsaruntip, E. Yenilmez, X. Tang, Q. Wang, Y.-L. Chang, H. Dai, An Investigation of the Mechanisms of Electronic Sensing of Protein Adsorption on Carbon Nanotube Devices, J Am Chem Soc, 126 (2004) 1563-1568. 
[117] X. Tang, S. Bansaruntip, N. Nakayama, E. Yenilmez, Y.-l. Chang, Q. Wang, Carbon Nanotube DNA Sensor and Sensing Mechanism, Nano Lett, 6 (2006) 1632-1636.

[118] I. Heller, A.M. Janssens, J. Männik, E.D. Minot, S.G. Lemay, C. Dekker, Identifying the Mechanism of Biosensing with Carbon Nanotube Transistors, Nano Lett, 8 (2008) 591-595.

[119] D. Wei, Y. Liu, Controllable synthesis of graphene and its applications, Adv Mater, 22 (2010) 3225-3241.

[120] Y.X. Huang, X.C. Dong, Y.X. Liu, L.J. Li, P. Chen, Graphene-based biosensors for detection of bacteria and their metabolic activities, J Mater Chem, 21 (2011) 12358-12362.

[121] D.S. Hecht, R.J.A. Ramirez, M. Briman, E. Artukovic, K.S. Chichak, J.F. Stoddart, G. Grüner, Bioinspired Detection of Light Using a Porphyrin-Sensitized Single-Wall Nanotube Field Effect Transistor, Nano Lett, 6 (2006) 2031-2036.

[122] A. Maroto, K. Balasubramanian, M. Burghard, K. Kern, Functionalized metallic carbon nanotube devices for $\mathrm{pH}$ sensing, Chemphyschem, 8 (2007) 220-223.

[123] K. Besteman, J.-O. Lee, F.G.M. Wiertz, H.A. Heering, C. Dekker, Enzyme-Coated Carbon Nanotubes as Single-Molecule Biosensors, Nano Lett, 3 (2003) 727-730.

[124] R.J. Chen, S. Bangsaruntip, K.A. Drouvalakis, N.W. Kam, M. Shim, Y. Li, W. Kim, P.J. Utz, H. Dai, Noncovalent functionalization of carbon nanotubes for highly specific electronic biosensors, Proc Natl Acad Sci USA, 100 (2003) 4984-4989.

[125] P. Attard, Electrolytes and the Electric Double Layer, Advances in Chemical Physics, John Wiley \& Sons, Inc. (2007) 1-159.

[126] S. Mao, G. Lu, K. Yu, Z. Bo, J. Chen, Specific protein detection using thermally reduced graphene oxide sheet decorated with gold nanoparticle-antibody conjugates, Adv Mater, 22 (2010) 3521-3526.

[127] B.M. Venkatesan, R. Bashir, Nanopore sensors for nucleic acid analysis, Nat Nanotechnol, 6 (2011) 615-624.

[128] M. Wanunu, T. Dadosh, V. Ray, J. Jin, L. McReynolds, M. Drndic, Rapid electronic detection of probe-specific microRNAs using thin nanopore sensors, Nat Nanotechnol, 5 (2010) 807-814.

[129] B. Song, G.F. Schneider, Q. Xu, G. Pandraud, C. Dekker, H. Zandbergen, Atomic-scale electronbeam sculpting of near-defect-free graphene nanostructures, Nano Lett, 11 (2011) 2247-2250.

[130] L. Tang, Y. Wang, J. Li, The graphene/nucleic acid nanobiointerface, Chem Soc Rev, 44 (2015) 6954-6980.

[131] G.F. Schneider, Q. Xu, S. Hage, S. Luik, J.N.H. Spoor, S. Malladi, H. Zandbergen, C. Dekker, Tailoring the hydrophobicity of graphene for its use as nanopores for DNA translocation, Nat Commun, 4 (2013).

[132] J.T. Robinson, M. Jorgolli, A.K. Shalek, M.-H. Yoon, R.S. Gertner, H. Park, Vertical nanowire electrode arrays as a scalable platform for intracellular interfacing to neuronal circuits, Nat Nanotechnol, 7 (2012) 180-184.

[133] L. Berdondini, K. Imfeld, A. Maccione, M. Tedesco, S. Neukom, M. Koudelka-Hep, S. Martinoia, Active pixel sensor array for high spatio-temporal resolution electrophysiological recordings from single cell to large scale neuronal networks, Lab Chip, 9 (2009) 2644-2651.

[134] M. Hutzler, A. Lambacher, B. Eversmann, M. Jenkner, R. Thewes, P. Fromherz, High-resolution multitransistor array recording of electrical field potentials in cultured brain slices, J Neurophysiol, 96 (2006) 1638-1645.

[135] L.R. Hochberg, M.D. Serruya, G.M. Friehs, J.A. Mukand, M. Saleh, A.H. Caplan, A. Branner, D. Chen, R.D. Penn, J.P. Donoghue, Neuronal ensemble control of prosthetic devices by a human with tetraplegia, Nature, 442 (2006) 164-171.

[136] A.B. Schwartz, Cortical neural prosthetics, Annu Rev Neurosci, 27 (2004) 487-507.

[137] J. Bai, X. Duan, Y. Huang, Rational Fabrication of Graphene Nanoribbons Using a Nanowire Etch Mask, Nano Lett, 9 (2009) 2083-2087.

[138] J. Bai, X. Zhong, S. Jiang, Y. Huang, X. Duan, Graphene nanomesh, Nat Nanotechnol, 5 (2010) 
190-194.

[139] M.Y. Han, B. Ozyilmaz, Y. Zhang, P. Kim, Energy band-gap engineering of graphene nanoribbons, Phys Rev Lett, 98 (2007) 206805.

[140] T. Kim, H. Kim, S.W. Kwon, Y. Kim, W.K. Park, D.H. Yoon, A.R. Jang, H.S. Shin, K.S. Suh, W.S. Yang, Large-Scale Graphene Micropatterns via Self-Assembly-Mediated Process for Flexible Device Application, Nano Lett, 12 (2012) 743-748.

[141] A. Sinitskii, J.M. Tour, Patterning Graphene through the Self-Assembled Templates: Toward Periodic Two-Dimensional Graphene Nanostructures with Semiconductor Properties, J Am Chem Soc, 132 (2010) 14730-14732.

[142] Z. Chen, Y.-M. Lin, M.J. Rooks, P. Avouris, Graphene nano-ribbon electronics, Physica E Low Dimens Syst Nanostruct, 40 (2007) 228-232.

[143] Ç.Ö. Girit, J.C. Meyer, R. Erni, M.D. Rossell, C. Kisielowski, L. Yang, C.-H. Park, M.F. Crommie, M.L. Cohen, S.G. Louie, A. Zettl, Graphene at the Edge: Stability and Dynamics, Science, 323 (2009) 1705-1708.

[144] J.Y. Huang, F. Ding, B.I. Yakobson, P. Lu, L. Qi, J. Li, In situ observation of graphene sublimation and multi-layer edge reconstructions, Proc Natl Acad Sci USA, 106 (2009) 1010310108.

[145] J.C. Meyer, C.O. Girit, M.F. Crommie, A. Zettl, Hydrocarbon lithography on graphene membranes, Appl Phys Lett, 92 (2008) 123110.

[146] Z. Shi, R. Yang, L. Zhang, Y. Wang, D. Liu, D. Shi, E. Wang, G. Zhang, Patterning graphene with zigzag edges by self-aligned anisotropic etching, Adv Mater, 23 (2011) 3061-3065.

[147] B. Song, G.F. Schneider, Q. Xu, G. Pandraud, C. Dekker, H. Zandbergen, Atomic-Scale ElectronBeam Sculpting of Near-Defect-Free Graphene Nanostructures, Nano Lett, 11 (2011) 2247-2250.

[148] M.C. Lemme, D.C. Bell, J.R. Williams, L.A. Stern, B.W.H. Baugher, P. Jarillo-Herrero, C.M. Marcus, Etching of Graphene Devices with a Helium Ion Beam, ACS Nano, 3 (2009) 2674-2676.

[149] V. Strong, S. Dubin, M.F. El-Kady, A. Lech, Y. Wang, B.H. Weiller, R.B. Kaner, Patterning and Electronic Tuning of Laser Scribed Graphene for Flexible All-Carbon Devices, ACS Nano, 6 (2012) 1395-1403.

[150] Y.-L. Zhang, Q.-D. Chen, H. Xia, H.-B. Sun, Designable 3D nanofabrication by femtosecond laser direct writing, Nano Today, 5 (2010) 435-448.

[151] J. Cai, P. Ruffieux, R. Jaafar, M. Bieri, T. Braun, S. Blankenburg, M. Muoth, A.P. Seitsonen, M. Saleh, X. Feng, K. Mullen, R. Fasel, Atomically precise bottom-up fabrication of graphene nanoribbons, Nature, 466 (2010) 470-473.

[152] M. Sprinkle, M. Ruan, Y. Hu, J. Hankinson, M. Rubio-Roy, B. Zhang, X. Wu, C. Berger, W.A. de Heer, Scalable templated growth of graphene nanoribbons on SiC, Nat Nanotechnol, 5 (2010) 727-731.

[153] M. Treier, C.A. Pignedoli, T. Laino, R. Rieger, K. Mullen, D. Passerone, R. Fasel, Surfaceassisted cyclodehydrogenation provides a synthetic route towards easily processable and chemically tailored nanographenes, Nat Chem, 3 (2011) 61-67.

[154] H. Wang, X. Wang, X. Li, H. Dai, Chemical self-assembly of graphene sheets, Nano Research, 2 (2009) 336-342.

[155] X. Yang, X. Dou, A. Rouhanipour, L. Zhi, H.J. Räder, K. Müllen, Two-Dimensional Graphene Nanoribbons, J Am Chem Soc, 130 (2008) 4216-4217.

[156] C. Vieu, F. Carcenac, A. Pépin, Y. Chen, M. Mejias, A. Lebib, L. Manin-Ferlazzo, L. Couraud, H. Launois, Electron beam lithography: resolution limits and applications, Appl Surf Sci, 164 (2000) 111-117.

[157] Y. Li, L. Huang, M. Zhong, Z. Wei, J. Li, An Efficient and Low-Cost Photolithographic-PatternTransfer Technique to Fabricate Electrode Arrays for Micro-/Nanoelectronics, Adv Mater Technol, (2016).

[158] J. Benschop, V. Banine, S. Lok, E. Loopstra, Extreme ultraviolet lithography: Status and 
prospects, J Vac Sci Technol B Microelectron Nanometer Struct, 26 (2008) 2204-2207.

[159] G.J. Burger, E.J.T. Smulders, J.W. Berenschot, T.S.J. Lammerink, J.H.J. Fluitman, S. Imai, Highresolution shadow-mask patterning in deep holes and its application to an electrical wafer feedthrough, Sens Actuator A: Physical, 54 (1996) 669-673.

[160] M. Elbahri, S.K. Rudra, S. Wille, S. Jebril, M. Scharnberg, D. Paretkar, R. Kunz, H. Rui, A. Biswas, R. Adelung, Employing Thin-Film Delamination for the Formation of Shadow Masks for Nanostructure Fabrication, Adv Mater, 18 (2006) 1059-1062.

[161] R.F.W. Pease, Electron beam lithography, Contemp Phys, 22 (2006) 265-290.

[162] R. Stine, J.T. Robinson, P.E. Sheehan, C.R. Tamanaha, Real-time DNA detection using reduced graphene oxide field effect transistors, Adv Mater, 22 (2010) 5297-5300.

[163] A. Allain, J. Kang, K. Banerjee, A. Kis, Electrical contacts to two-dimensional semiconductors, Nat Mater, 14 (2015) 1195-1205.

[164] Y.J. Shin, Y. Wang, H. Huang, G. Kalon, A.T. Wee, Z. Shen, C.S. Bhatia, H. Yang, Surfaceenergy engineering of graphene, Langmuir, 26 (2010) 3798-3802.

[165] Y. Ohno, K. Maehashi, Y. Yamashiro, K. Matsumoto, Electrolyte-Gated Graphene Field-Effect Transistors for Detecting pH and Protein Adsorption, Nano Lett, 9 (2009) 3318-3322.

[166] N.C.S. Vieira, J. Borme, J. G. Machado, F. Cerqueira, P.P. Freitas, V. Zucolotto, N.M.R. Peres, P. Alpuim, Graphene field-effect transistor array with integrated electrolytic gates scaled to $200 \mathrm{~mm}$, J Phys Condens Matter, 28 (2016) 085302.

[167] V. Georgakilas, M. Otyepka, A.B. Bourlinos, V. Chandra, N. Kim, K.C. Kemp, P. Hobza, R. Zboril, K.S. Kim, Functionalization of graphene: covalent and non-covalent approaches, derivatives and applications, Chem Rev, 112 (2012) 6156-6214.

[168] Y. Huang, X. Dong, Y. Shi, C.M. Li, L.J. Li, P. Chen, Nanoelectronic biosensors based on CVD grown graphene, Nanoscale, 2 (2010) 1485-1488.

[169] Q.H. Wang, M.C. Hersam, Room-temperature molecular-resolution characterization of selfassembled organic monolayers on epitaxial graphene, Nat Chem, 1 (2009) 206-211.

[170] S. Mao, K. Yu, G. Lu, J. Chen, Highly sensitive protein sensor based on thermally-reduced graphene oxide field-effect transistor, Nano Research, 4 (2011) 921-930.

[171] Y. Liu, D.S. Yu, C. Zeng, Z.C. Miao, L.M. Dai, Biocompatible Graphene Oxide-Based Glucose Biosensors, Langmuir, 26 (2010) 6158-6160.

[172] Y. Wan, Y. Wang, J.J. Wu, D. Zhag, Graphene Oxide Sheet-Mediated Silver Enhancement for Application to Electrochemical Biosensors, Anal Chem, 83 (2011) 648-653.

[173] P. Wu, Q. Shao, Y. Hu, J. Jin, Y. Yin, H. Zhang, C. Cai, Direct electrochemistry of glucose oxidase assembled on graphene and application to glucose detection, Electrochim Acta, 55 (2010) 8606-8614.

[174] K. Zhou, Y. Zhu, X. Yang, C. Li, Electrocatalytic Oxidation of Glucose by the Glucose Oxidase Immobilized in Graphene-Au-Nafion Biocomposite, Electroanal, 22 (2010) 259-264.

[175] J. Li, S.J. Guo, Y.M. Zhai, E.K. Wang, High-sensitivity determination of lead and cadmium based on the Nafion-graphene composite film, Anal Chim Acta, 649 (2009) 196-201.

[176] N. Ruecha, R. Rangkupan, N. Rodthongkum, O. Chailapakul, Novel paper-based cholesterol biosensor using graphene/polyvinylpyrrolidone/polyaniline nanocomposite, Biosens Bioelectron, 52 (2014) 13-19.

[177] R. Roldan, A. Castellanos-Gomez, E. Cappelluti, F. Guinea, Strain engineering in semiconducting two-dimensional crystals, J Phys Condens Matter, 27 (2015) 313201.

[178] G. Xu, J. Abbott, L. Qin, K.Y.M. Yeung, Y. Song, H. Yoon, J. Kong, D. Ham, Electrophoretic and field-effect graphene for all-electrical DNA array technology, Nat Commun, 5 (2014).

[179] G.S. Kulkarni, K. Reddy, Z. Zhong, X. Fan, Graphene nanoelectronic heterodyne sensor for rapid and sensitive vapour detection, Nat Commun, 5 (2014) 4376.

[180] S.H. Choi, Y.L. Kim, K.M. Byun, Graphene-on-silver substrates for sensitive surface plasmon resonance imaging biosensors, Opt Express, 19 (2011) 458-466. 
[181] M.D. Fischbein, M. Drndić, Electron beam nanosculpting of suspended graphene sheets, Appl Phys Lett, 93 (2008) 113107.

[182] A. Singer, M. Wanunu, W. Morrison, H. Kuhn, M. Frank-Kamenetskii, A. Meller, Nanopore based sequence specific detection of duplex DNA for genomic profiling, Nano Lett, 10 (2010) 738-742.

[183] S.K. Min, W.Y. Kim, Y. Cho, K.S. Kim, Fast DNA sequencing with a graphene-based nanochannel device, Nat Nanotechnol, 6 (2011) 162-165.

[184] S.J. Ling, C.X. Li, J. Adamcik, S.H. Wang, Z.Z. Shao, X. Chen, R. Mezzenga, Directed Growth of Silk Nanofibrils on Graphene and Their Hybrid Nanocomposites, ACS Macro Lett, 3 (2014) 146-152.

[185] T.-i. Kim, J.G. McCall, Y.H. Jung, X. Huang, E.R. Siuda, Y. Li, J. Song, Y.M. Song, H.A. Pao, R.-H. Kim, C. Lu, S.D. Lee, I.-S. Song, G. Shin, R. Al-Hasani, S. Kim, M.P. Tan, Y. Huang, F.G. Omenetto, J.A. Rogers, M.R. Bruchas, Injectable, Cellular-Scale Optoelectronics with Applications for Wireless Optogenetics, Science, 340 (2013) 211-216.

[186] E. Pastrana, Optogenetics: controlling cell function with light, Nat Meth, 8 (2011) 24-25.

[187] L.H. Hess, M. Jansen, V. Maybeck, M.V. Hauf, M. Seifert, M. Stutzmann, I.D. Sharp, A. Offenhausser, J.A. Garrido, Graphene transistor arrays for recording action potentials from electrogenic cells, Adv Mater, 23 (2011) 5045-5049, 4968.

[188] H.J. Conley, B. Wang, J.I. Ziegler, R.F. Haglund, Jr., S.T. Pantelides, K.I. Bolotin, Bandgap engineering of strained monolayer and bilayer $\mathrm{MoS}_{2}$, Nano Lett, 13 (2013) 3626-3630.

[189] T. Cohen-Karni, B.P. Timko, L.E. Weiss, C.M. Lieber, Flexible electrical recording from cells using nanowire transistor arrays, Proc Natl Acad Sci USA, 106 (2009) 7309-7313.

[190] M.A. Bissett, M. Tsuji, H. Ago, Strain engineering the properties of graphene and other twodimensional crystals, Phys Chem Chem Phys, 16 (2014) 11124-11138.

[191] Y. Wang, R. Yang, Z.W. Shi, L.C. Zhang, D.X. Shi, E. Wang, G.Y. Zhang, Super-Elastic Graphene Ripples for Flexible Strain Sensors, ACS Nano, 5 (2011) 3645-3650.

[192] M.C. Wang, S. Chun, R.S. Han, A. Ashraf, P. Kang, S. Nam, Heterogeneous, three-dimensional texturing of graphene, Nano Lett, 15 (2015) 1829-1835.

[193] Z. Wen, X. Wang, S. Mao, Z. Bo, H. Kim, S. Cui, G. Lu, X. Feng, J. Chen, Crumpled nitrogendoped graphene nanosheets with ultrahigh pore volume for high-performance supercapacitor, Adv Mater, 24 (2012) 5610-5616.

[194] J. Leem, M.C. Wang, P. Kang, S. Nam, Mechanically Self-Assembled, Three-Dimensional Graphene-Gold Hybrid Nanostructures for Advanced Nanoplasmonic Sensors, Nano Lett, 15 (2015) 7684-7690.

[195] J. Feng, X. Qian, C.-W. Huang, J. Li, Strain-engineered artificial atom as a broad-spectrum solar energy funnel, Nat Photon, 6 (2012) 866-872.

[196] K. He, C. Poole, K.F. Mak, J. Shan, Experimental demonstration of continuous electronic structure tuning via strain in atomically thin $\mathrm{MoS}_{2}$, Nano Lett, 13 (2013) 2931-2936.

[197] Y.Y. Hui, X. Liu, W. Jie, N.Y. Chan, J. Hao, Y.-T. Hsu, L.-J. Li, W. Guo, S.P. Lau, Exceptional Tunability of Band Energy in a Compressively Strained Trilayer $\mathrm{MoS}_{2}$ Sheet, ACS Nano, 7 (2013) 7126-7131.

[198] P. Lu, X. Wu, W. Guo, X.C. Zeng, Strain-dependent electronic and magnetic properties of $\mathrm{MoS}_{2}$ monolayer, bilayer, nanoribbons and nanotubes, Phys Chem Chem Phys, 14 (2012) 13035-13040.

[199] A.V. Thomas, B.C. Andow, S. Suresh, O. Eksik, J. Yin, A.H. Dyson, N. Koratkar, Controlled Crumpling of Graphene Oxide Films for Tunable Optical Transmittance, Adv Mater, 27 (2015) 3222-3222.

[200] J. Zang, S. Ryu, N. Pugno, Q. Wang, Q. Tu, M.J. Buehler, X. Zhao, Multifunctionality and control of the crumpling and unfolding of large-area graphene, Nat Mater, 12 (2013) 321-325.

[201] J. Choi, H.J. Kim, M.C. Wang, J. Leem, W.P. King, S. Nam, Three-Dimensional Integration of Graphene via Swelling, Shrinking, and Adaptation, Nano Lett, 15 (2015) 4525-4531. 
[202] R. Hooper, A.A. Prinz, Dynamic Clamp in Biomimetic and Biohybrid Living-Hardware Systems, Biohybrid Systems, Wiley-VCH Verlag GmbH \& Co. KGaA (2011) 77-94.

[203] A. Ashraf, Y. Wu, M.C. Wang, N.R. Aluru, S.A. Dastgheib, S. Nam, Spectroscopic investigation of the wettability of multilayer graphene using highly ordered pyrolytic graphite as a model material, Langmuir, 30 (2014) 12827-12836.

[204] A. Zhang, C.M. Lieber, Nano-Bioelectronics, Chem Rev, 116 (2016) 215-257.

[205] A.A. Balandin, Low-frequency $1 / f$ noise in graphene devices, Nat Nanotechnol, 8 (2013) 549-555.

[206] X. Xie, D. Sarkar, W. Liu, J. Kang, O. Marinov, M.J. Deen, K. Banerjee, Low-Frequency Noise in Bilayer $\mathrm{MoS}_{2}$ Transistor, ACS Nano, 8 (2014) 5633-5640.

[207] K. Kang, S. Xie, L. Huang, Y. Han, P.Y. Huang, K.F. Mak, C.J. Kim, D. Muller, J. Park, Highmobility three-atom-thick semiconducting films with wafer-scale homogeneity, Nature, 520 (2015) 656-660. 
